\title{
Arylnaphthalene lactones: structures and pharmacological potentials
}

\author{
Soyoung Park $\cdot$ Seungsu Kim $\cdot$ Dongyun Shin $\mathbb{B}$
}

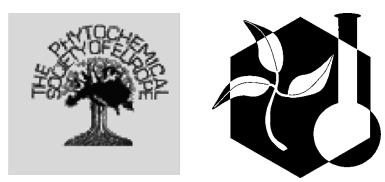

Received: 11 July 2020/Accepted: 17 December 2020/Published online: 10 March 2021

(C) The Author(s) 2021

\begin{abstract}
Natural arylnaphthalene lactones are representative lignans that are found in various dietary and medicinal plants. Their unique structural features and significant pharmacological activity have attracted considerable attention from both synthetic and medicinal chemists. Owing to their unique structural features such as relative rigid tetracyclic skeleton, structural diversity of more than five substituents, and no chiral center, arylnaphthalene lactones are recognized as a valuable scaffold for drug discovery, in addition to their significant pharmacological activities. This review covers the structures and isolation of all naturally occurring arylnaphthalene lactone congeners reported. Based on the aryl substituents, they were categorized as Type I and Type II and further classified according to the oxidation state of the ring and glycosylation level. Special attention has been paid to natural arylnaphthalene lactones owing to their broad spectrum of biological activities such as cytotoxic, antiplatelet, antiviral, anti-HIV, antifungal, neuroprotective, and anti-inflammatory properties. All the products were reorganized based
\end{abstract}

Soyoung Park and Seungsu Kim have contributed equally to this work.

S. Park · S. Kim · D. Shin $(\square)$

College of Pharmacy, Gachon University, 191

Hambakmoe-ro, Yeonsu-gu, Incheon 21936, Korea

e-mail: dyshin@gachon.ac.kr on their biological activities, and selected data are presented.

Keywords Arylnaphthalene lactones · Lignan · Medicinal · Natural · Activity

\section{Introduction}

Arylnaphthalene lignan lactones are naturally occurring fused tricyclic naphthalene lactones with aryl substituents. Structurally, arylnaphthalene lignan lactones consist of two arylpropanoid units and are classified as Type I and Type II (Fig. 1) based on the relative position of lactone and the aryl substituents (Teponno et al. 2016). Approximately 59 natural arylnaphthalene lignan lactones and their glycosylated congeners have been isolated from various dietary and medicinal plants and structurally elucidated. The broad spectrum of their pharmacological benefits has also been reported such as antiproliferative, antiplatelet aggregation, antiviral, antifungal, neuroprotective, and anti-inflammatory activities.

The unique structural features as well as promising bioactivities of arylnaphthalene lactones have drawn considerable attention from synthetic chemists. Since the first synthesis of an arylnaphthalene lignan lactone skeleton in 1895 by the Bucher group (Michael and Bucher 1895) via the condensation of arylpropiolic 
Fig. 1 Structure of arylnaphthalene lactones

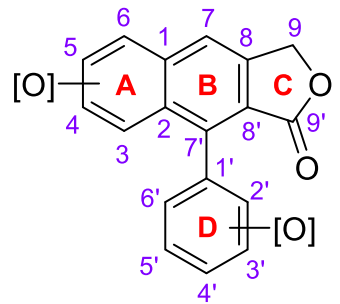

Type I

aryInaphthalene lactone<smiles>O=C1OCc2c1cc1ccc(Cl)cc1c2-c1ccccc1Cl</smiles>

Type II

arylnaphthalene lactone acids, various synthetic approaches for arylnaphthalene lignan lactones have been designed and applied successfully. Major synthetic approaches include the intramolecular Diels-Alder reaction for the construction of an arylnaphthalene lactone from arylpropiolic anhydride (Brown and Stevenson 1964, 1965; Maclean and Stevenson 1966; Block and Stevenson 1971; Holmes and Stevenson 1970, 1971; Stevenson and Holmes 1971; Stevenson and Block 1971; Block and Stevenson 1973; Stevenson and Weber 1989, 1991; Anastas and Stevenson 1991; Park et al. 2014). Intermolecular Diels-Alder approaches were also investigated using isobenzofurans and acetylenedicarboxylate (de Silva et al. 1980; Plaumann et al. 1980). Other valuable synthetic methodologies utilizing key reactions such as the Blaise reaction-intramolecular $[4+2]$ reaction $(\mathrm{He}$ et al. 2012), Garratt-Braverman cyclization (Block and Stevenson, 1971, 1973; Arnold et al. 1973; Yamamoto et al. 2015), benzoin condensation-thermal cyclization (Hayat et al. 2015a, b), and transitionmetal catalyzed synthesis (Park et al. 2020) have been reported.

Although the isolation and chemistry of natural lignan products has been broadly reviewed (Teponno et al. 2016; Li et al. 2020), a focused and comprehensive review on the structures and beneficial biological activities of natural arylnaphthalene lignan lactones has not been published. The purpose of this review is to provide a compilation of naturally occurring arylnaphthalene lignan lactones in terms of structure, isolation, and pharmacological activity.

\section{Structures and isolation}

Arylnaphthalene lignan lactones are found in a variety of dietary and medicinal herbs including Phyllanthus,
Justicia, Haplophyllum, and Cleistanthus. Arylnaphthalene lignan lactones are classified into two types based on their structures, 1-phenyl-2-hydroxymethylnaphthalene-2-carboxylic acid lactone (Type I) and 1-phenyl-3-hydroxymethylnaphthalene-3-carboxylic acid lactone (Type II). To provide a visual reference guide for each compound and to present an overview of the biological activities of arylnaphthalene lactones, all naturally occurring derivatives are classified by their types in Figs. 2 and 3. In Fig. 2, Type I compounds are presented, and they can be divided into three groups as 7-unsubstituted, 7-oxygenated-, and 7-O-glycosylated arylnaphthalene lactones. The first subclass of arylnaphthalene lactones includes four oxygenated congeners: justicidin B (1) (Gözler et al. 1984; Luo et al. 2014; Rao et al. 2006; Batsuren et al. 1981; Batirov et al. 1981; Lin et al. 1995; Gertsch et al. 2003; Hesse et al. 1992; Hemmati et al. 2016; Mohagheghzadeh et al. 2002) taiwanin C (2) (Yang et al. 2006; Anjaneyulu et al. 1981; Ban et al. 2002; Sastry and Rao 1983), daurinol (4) (Batsuren et al. 1981; Hesse et al. 1992), isodaurinol (5) (Hesse et al. 1992), and chinensin (7) (Ghosal et al. 1974; Cow et al. 2000), which are basic forms of natural arylnaphthalene lactones and only differ in the substituents on the alcohols. Several compounds that are further oxygenated at ring $\mathrm{A}$ or ring $\mathrm{D}$ such as deoxydehydropodophyllotoxin (8) (Novelo et al. 1993), dehydro- $\beta$-peltatin methyl ether (11) (Novelo et al. 1993), phyllamyricin C (12) (Rao et al. 2006; Lin et al. 1995), koelreuterin-1 (6) (Song et al. 1994), and justicidin H (3) (Yang et al. 2006) have been identified. Justicidinoside C (9) (Asano et al. 1996), which is the mono-glycosylated product of justicidin $\mathrm{C}$, has also been isolated. 9-Hydroxy or 9-methoxy naphthalene lactones such as piscatorin (10) (Windayani et al. 2014; Gertsch et al. 2003), phyllamyricin 


\section{A. Type I arylnaphthalene lactones}<smiles>COc1cc2cc3c(c(-c4ccc5c(c4)OCO5)c2cc1OC)C(=O)OC3</smiles>

1. Justicidin B

Gozler, Gozler et al. 1984

H. buxbaumii

Luo, Hu et al. 2014

J. procumbens

Rao, Fang et al. 2006

$P$. polyphyllus

Batsuren, Batirov et al. 1981

H. dauricum<smiles>COc1cc2cc3c(c(-c4cc5c(cc4O)OCO5)c2cc1OC)C(=O)OC3</smiles>

3. Justicidin $\mathrm{H}$ (6'-Hydroxy Justicidin B) Yang, Wu et al. 2006

J. procumbens<smiles>COc1ccc(-c2c3c(cc4cc5c(cc24)OCO5)COC3=O)cc1OC</smiles>

7. Chinensin

Ghosal, Chauhan et al. 1974 $P$. chinensis<smiles>COc1cc(-c2c3c(cc4cc(OC)c5c(c24)OCO5)COC3=O)cc(OC)c1OC</smiles>

11. Dehydro- $\beta$-peltatin methyl ether

Novelo, Cruz et al. 1993

$H$. verticillata
Lin, Lee et al. 1995

$P$. myrtifolius

Gertsch, Tobler et al. 2003

$P$. piscatorum

Hesse, Gozler et al. 1992

H. cappadocicum<smiles>O=C1OCc2cc3cc4c(cc3c(-c3ccc5c(c3)OCO5)c21)OCO4</smiles>

2. Taiwanin $\mathrm{C}$

Yang, Wu et al. 2006

J. procumbens

Anjaneyulu, Ramaiah

et al. 1981

C. collinus<smiles>COc1cc2c(-c3ccc4c(c3)OCO4)c3c(cc2cc1O)COC3=O</smiles>

4. Daurinol

(5'-Demethyljusticidin B)

Batsuren, Batirov et al. 1981

H. dauricum

Hesse, Gozler et al. 1992

H. cappadocicum<smiles>COc1cc(-c2c3c(cc4cc5c(cc24)OCO5)COC3=O)cc(OC)c1OC</smiles><smiles>COc1cc2cc3c(c(-c4ccc5c(c4)OCO5)c2cc1O)C(=O)OC3</smiles>

5. Isodaurinol Hesse, Gozler et al. 1992 H. cappadocium<smiles>COc1cc(-c2c3c(cc4cc5c(cc24)OCO5)COC3=O)cc2c1OCO2</smiles>

6. Koelreuterin-1 Song, Zhang et al. 1994 K. henryi<smiles>COc1cc2cc3c(c(-c4cc5c(cc4O[C@@H]4O[C@H](CO)[C@@H](O)[C@H](O)[C@H]4O)OCO5)c2cc1OC)C(=O)OC3</smiles>

9. Justicidinoside $C$ Asano, Chiba et al. 1996 J. procumbens<smiles>COc1cc2c(-c3ccc4c(c3)OCO4)c3c(cc2c(OC)c1OC)C(=O)OC3OC</smiles>

13. Phyllamyricin $D$ Lin, Lee et al. 1995 P. myrtifolius<smiles>COc1cc2c(OC)cc3cc4c(c(-c5ccc6c(c5)OCO6)c3c2cc1OC)C(=O)OC4O</smiles>

10. Piscatorin Windayani 2014 P. myrtifolius Gertsch, Tobler et al. 2003 $P$. piscatorum<smiles>COc1cc2cc3c(c(-c4ccc5c(c4)OCO5)c2cc1OC)C(=O)OC3OC</smiles>

14. Phyllamyricin $E$ Lin, Lee et al. 1995 P. myrtifolius
Rao, Fang et al. 2006

P. polyphyllus

Lin, Lee et al. 1995

P. myrtifolius

Fig. 2 Structure and isolation of Type I arylnaphthalene lactones 
<smiles>COc1cc2cc3c(c(-c4ccc(OC5OC(CO)[C@@H](O)[C@H](O)C5O)c(O)c4)c2cc1OC)C(=O)OC3</smiles>

15. Procumbenoside $L$ Jin et al. 2017 J. procumbens<smiles>COc1cc(-c2c3c(cc4cc5c(cc24)OCO5)COC3=O)ccc1O</smiles>

16. 5-(4-Hydroxy-3methoxyphenyl)furo[3', $\left.4^{\prime}: 6,7\right]$ na phtho[2,3-d]-1,3-dioxol-6(8H)one

Liu et al 2008

B. marginatum<smiles>COc1ccc2cc3c(c(-c4ccc5c(c4)OCO5)c2c1OC)C(=O)OC3</smiles>

17. 3,4-Dimethoxy-3',4'methylenedioxy-2,7'cycloligna-7,7'-dieno-9,9'-

lactone

Mohagheghsadeh et al. 2002

L. austriacum

\section{B. 7-Oxygenated Type I arylnaphthalene lactones}<smiles>COc1cc2c(O)c3c(c(-c4ccc5c(c4)OCO5)c2cc1OC)C(=O)OC3</smiles>

18. Diphyllin

Burden, Crombie et al. 1969 Anjaneyulu, Ramaiah et al. 1981 H. tuberculatum

Chen, Hsin et al. 1996

J. procumbens

Rao, Fang et al. 2006

$P$. polyphyllus

Gozler, Gozler et al. 1984

H. Vulcanicum<smiles>COc1cc2c(O)c3c(c(-c4ccc5c(c4)OCO5)c2cc1OC)C(=O)OC3OC</smiles>

21. Cleistanone

Ramesh, Ravindranath et al. 2003

C. collinus<smiles>O=C1OCc2c1c(-c1cc3c(cc1O)OCO3)c1c3c(ccc1c2O)OCO3</smiles>

25. 2'-Hydroxy-justirumalin Rezanka et al. 2009 A. mollis

$P$. oligospermus Evcim, Gozler et al. 1986 H. myrtifolium

26. Justicidin A Burden, Crombie et al. 1969 H. tuberculatum<smiles>COc1ccc(-c2c3c(c(O)c4cc5c(cc24)OCO5)C(=O)OC3)cc1OC</smiles>

19. Chinensinaphthol (Isodiphyllin) Chen, Hsin et al. 1996 J. procumbens Day, Chiu et al. 1999 J. ciliata Ghosal, Chauhan et al. 1974 $P$. chinensis<smiles>COc1cc(-c2c3c(c(O)c4c(OC)c5c(cc24)OCO5)COC3=O)cc(OC)c1OC</smiles>

23. 5-Methoxydehydropodophyllotoxin Novelo, Cruz et al. 1993 H. verticillata<smiles>COc1cc2c(OC)c3c(c(-c4ccc5c(c4)OCO5)c2cc1OC)C(=O)OC3</smiles>
Day, Lin et al. 2002

J. procumbens

Wu and Wu 2006

$P$. oligospermus

Lin, Lee et al. 1995

P. myrtifolius<smiles>O=C1OCc2c1c(-c1ccc3c(c1)OCO3)c1cc3c(cc1c2O)OCO3</smiles>

20. Taiwanin E

Chen, Hsin et al. 1996

J. procumbens

Anjaneyulu, Ramaiah et al. 1981 C. collinus

Wang, Tseng et al. 2014

E. trifoliatus

Susplugas, Hung et al. 2005

J. patentiflora $\mathrm{OH}$<smiles>COc1cc(-c2c3c(c(O)c4cc5c(cc24)OCO5)COC3=O)cc(OC)c1OC</smiles>

24. Dehydropodophyllotoxin Novelo, Cruz et al. 1993 $H$. verticillata<smiles>COc1cc2c(OC)c3c(c(-c4cc5c(cc4O)OCO5)c2cc1OC)C(=O)OC3</smiles>

27. 6'-Hydroxy Justicidin A Yang, Wu et al. 2006 J. procumbens

Fig. 2 continued 
<smiles>COc1cc(-c2c3c(c(OC)c4cc(OC)c(OC)cc24)COC3=O)ccc1O</smiles>

28. Cilinaphthalide $A$ Day, Chiu et al. 1999 J. ciliata<smiles>COc1c2c(c(-c3ccc4c(c3)OCO4)c3cc4c(cc13)OCO4)C(=O)OC2</smiles>

32. Justicidin $F$ (Taiwanin E methyl ether) Chen, Hsin et al. 1996 J. procumbens Day, Chiu et al. 1999 J. ciliata<smiles>COc1cc2c(-c3ccc4c(c3)OCO4)c3c(c(OC)c2c(O)c1OC)COC3=O</smiles>

36. 5-Hydroxyjusticidin A Tian, Hao et al. 2006 M. patentiflora<smiles>COc1cc(-c2c3c(c(OC)c4cc5c(cc24)OCO5)C(=O)OC3)cc(OC)c1O</smiles>

40. 4'-O-demethyl-7-Omethyldehydropodophyl lotoxin

Wei et al. 2018

H. nymphaeifolia<smiles>COc1ccc(-c2c3c(c(OC)c4cc(OC)c(OC)cc24)COC3=O)cc1OC</smiles>

29. Cilinaphthalide $B$ Weng, Ko et al. 2004 J. procumbens

Day, Chiu et al. 1999 J. ciliata<smiles>COc1cc2c(OC)c3c(c(-c4ccc5c(c4)OCO5)c2cc1OC)C(=O)OC3OC</smiles>

33. Justicidin $P$ Wang and Ripka 1983 J. extensa<smiles>COc1c2c(c(-c3ccc(O)c(O[C@@H]4O[C@H](CO)[C@@H](O)[C@H](O)[C@H]4O)c3)c3cc4c(cc13)OCO4)C(=O)OC2</smiles>

37. Justalakonin Kavitha et al. 2003 J. purpurea<smiles>COc1ccc(-c2c3c(c(OC)c4cc5c(cc24)OCO5)COC3=O)cc1OC</smiles>

30. Chinensinaphtholmethyl ether

Luo, Hu et al. 2014

J.procumbens<smiles>COc1cc2c(OC)c3c(c(-c4ccc5c(c4)OCO5)c2cc1O)C(=O)OC3</smiles>

31. Phyllanthusmin $A$ Wu and Wu 2006 $P$. oligospermus Ren et al., 2014 P.poilanei<smiles></smiles>

34. Justicinol Susplugas, Hung et al. 2005

J.patentiflora<smiles></smiles>

35. Justicidinoside $B$ Asano, Chiba et al. 1996 J. procumbens<smiles>COc1cc2c(OC)c3c(c(-c4ccc(OC5O[C@H](CO)[C@@H](O)[C@H](O)[C@H]5O)c(O)c4)c2cc1OC)C(=O)OC3</smiles>

38. Procumbenoside $K$ Jin et al. 2017 J. procumbens<smiles>COc1ccc(-c2c3c(c(OC)c4cc(OC)c(OC)cc24)COC3=O)cc1O</smiles>

39. Pronaphthalide A Jin et al. 2014 J. procumbens

Fig. 2 continued 


\section{7-0-glycosylated Type I arylnaphthalene lactones}<smiles>COc1cc2c(O[C@@H]3OC[C@](O)(CO)[C@H]3O)c3c(c(-c4ccc5c(c4)OCO5)c2cc1OC)C(=O)OC3</smiles>

41. Tuberculatin (diphyllin aposide) Susplugas, Hung et al. 2005 J. patentiflora

Innocenti, Puricelli et al. 2002 H. patavinum<smiles>COc1cc2c(O[C@@H]3OC[C@@H](OC)[C@H](OC)[C@H]3O)c3c(c(-c4ccc5c(c4)OCO5)c2cc1OC)C(=O)OC3</smiles>

45. Cleistanthin A Anjaneyulu, Ramaiah et al. 1981

C. collinus

Sastry and Rao 1983

C. patulus

Tuchinda et al., 2006

P. taxodiifolius<smiles>COc1cc2c(O[C@@H]3OC[C@H](OC(C)=O)[C@H](O)[C@H]3O)c3c(c(-c4ccc5c(c4)OCO5)c2cc1OC)C(=O)OC3</smiles>

49. Phyllanthusmin B Lin, Lee et al. 1995 P. myrtifolius

Ren, Lantvit et al. 2014

$P$. poilanei<smiles>COC[C@H]1O[C@@H](Oc2c3c(c(-c4ccc5c(c4)OCO5)c4cc(OC)c(OC)cc24)C(=O)OC3)[C@H](OC)[C@@H]1OC</smiles>

42. Cleistanthin $D$ Anjaneyulu, Ramaiah et al. 1981

C. collinus<smiles>COc1cc2c(O[C@@H]3OC[C@@H](OC)[C@H](OC)[C@H]3OC)c3c(c(-c4ccc5c(c4)OCO5)c2cc1OC)C(=O)OC3</smiles>

46. Cleistanthin A methyl ether

Tuchinda et al., 2006

$P$. taxodiifolius<smiles>COc1cc2c(O[C@@H]3OC[C@@H](O)[C@H](O)[C@H]3O)c3c(c(-c4ccc5c(c4)OCO5)c2cc1OC)C(=O)OC3</smiles>

50. Phyllanthusmin C Lin, Lee et al.1995 $P$. myrtifolius

Ren, Lantvit et al. 2014 P. poilanei<smiles>COc1cc2c(O[C@@H]3OC[C@](O)(COC(C)=O)[C@H]3O)c3c(c(-c4ccc5c(c4)OCO5)c2cc1OC)C(=O)OC3</smiles>

43. Diphyllin acetylapioside (mono-O-acetyl diphyllin) apioside Nukul, Abu Zarga et al. 1987 H. buxbaumii

Prieto, Giner et al. 2002 H. hispanicum<smiles>COc1cc2c(OC3O[C@H](CO)[C@@H](O)[C@H](O)[C@H]3O)c3c(c(-c4ccc5c(c4)OCO5)c2cc1OC)C(=O)OC3</smiles>

47. Cleistanthin $B$ (Diphyllin O-glycoside) Anjaneyulu, Ramaiah et al. 1981 C. collinus

Al-Abed, Sabri et al. 1990 H. buxbaumii

Ren, Lantvit et al. 2014

P. poilanei<smiles>COc1cc2c(O[C@@H]3OC[C@H](OC(C)=O)[C@H](OC(C)=O)[C@H]3O)c3c(c(-c4ccc5c(c4)OCO5)c2cc1OC)C(=O)OC3</smiles>

51. Phyllanthusmin $D$ Ren, Lantvit et al. 2014 $P$. poilanei<smiles>COc1cc2c(O[C@@H]3OC[C@](O)(CO)[C@H]3O)c3c(c(-c4ccc5c(c4)OCO5)c2cc1O)C(=O)OC3</smiles>

44. Haplomyrtoside Gozler, Gozler et al. 1996 H. Cappadocium<smiles>COc1cc2c(O[C@@H]3O[C@H](O)[C@@H](O)[C@H](O)[C@H]3O)c3c(c(-c4ccc5c(c4)OCO5)c2cc1OC)C(=O)OC3</smiles>

48. Mananthoside $A$ Chen, Liu et al. 2002 M. patentiflora<smiles>COc1cc2c(OC3OCC(O)[C@H](OC(C)=O)[C@H]3O)c3c(c(-c4ccc5c(c4)OCO5)c2cc1OC)C(=O)OC3</smiles>

52. Phyllanthusmin $E$ Ren, Lantvit et al. 2014 P. poilane $i$

Fig. 2 continued 
<smiles></smiles>

53. Procumbenoside $C$ Liu et al. 2008 J. procumbens<smiles>COc1cc2c(O[C@@H]3O[C@H](CO)[C@@H](O)[C@H](O)[C@H]3O)c3c(c(-c4ccc5c(c4)OCO5)c2cc1O)C(=O)OC3</smiles>

57. Procumbenoside I Jin et al. 2017 J. procumbens<smiles>COc1cc2c(O[C@@H]3O[C@H](C)[C@@H](OC(C)=O)[C@H](O)[C@H]3O)c3c(c(-c4ccc5c(c4)OCO5)c2cc1OC)C(=O)OC3</smiles>

61. 4"-O-acetylpatentiflorin B Susplugas, Hung et al. 2005 J. patentiflora<smiles>COc1ccc(-c2c3c(c(OC4OC(CO)C(O)[C@H](O)[C@H]4O)c4cc5c(cc24)OCO5)COC3=O)cc1OC</smiles>

54. Procumbenoside $D$ Liu et al. 2008 J. procumbens<smiles>COc1cc2c(O[C@@H]3OC(O)[C@H](O)[C@H]3OC)c3c(c(-c4ccc5c(c4)OCO5)c2cc1OC)C(=O)OC3</smiles>

58. Acutissimalignan $A$ Tuchinda et al. 2008 $P$. acutissima<smiles>COc1cc2c(OC3OC(C)C(O)[C@H](O)[C@H]3O)c3c(c(-c4ccc5c(c4)OCO5)c2cc1OC)C(=O)OC3</smiles>

55. Patentiflorin A Susplugas, Hung et al. 2005 J. patentiflora<smiles>COc1cc2c(OC)cc3c(O[C@@H]4O[C@H](CO)[C@@H](O)[C@H](O)[C@H]4O)c4c(c(-c5ccc6c(c5)OCO6)c3c2cc1OC)C(=O)OC4</smiles>

59. 7-O- $\beta$-D-glucopyranosyl justicidin $B$

Borges et al. 2018

$P$. brasiliensis<smiles>COc1cc2c(O[C@@H]3OC[C@](O)(CO)[C@H]3O[C@H]3OC[C@@H](O)[C@H](O)[C@H]3O)c3c(c(-c4ccc5c(c4)OCO5)c2cc1OC)C(=O)OC3</smiles>

62. Majidine Al-Abed, Sabri et al. 1990 H. buxbaumii Innocenti, Puricelli et al. 2002 H. patavinum<smiles>COc1cc2c(OC3OC[C@](O)(CO)[C@H]3OC3OC[C@@H](O)[C@H](O)[C@H]3O)c3c(c(-c4ccc5c(c4)OCO5)c2cc1OC)C(=O)OC3</smiles>

63. Procumbenoside $A$ Day, Lin et al. 2002 J. procumbens<smiles>COc1cc2c(O[C@@H]3O[C@H](C)[C@@H](O)[C@H](O)[C@H]3O)c3c(c(-c4ccc5c(c4)OCO5)c2cc1OC)C(=O)OC3</smiles>

56. Patentiflorin $B$ Susplugas, Hung et al. 2005 J. patentiflora<smiles>COc1cc(-c2c3c(c(OC4O[C@H](CO)[C@@H](O)[C@H](O)[C@H]4O)c4cc5c(cc24)OCO5)COC3=O)cc(OC)c1OC</smiles>

60. 7-O-( $\beta$-D-glucopyranosyl) dehydropodophyllotoxin

Liu et al. 2015

C. boivinianus<smiles>COc1cc2c(O[C@@H]3OC[C@](O)(CO)[C@H]3O[C@H]3O[C@H](CO)[C@@H](O)[C@H](O)[C@H]3O)c3c(c(-c4ccc5c(c4)OCO5)c2cc1OC)C(=O)OC3</smiles>

64. Procumbenoside $B$ Weng, Ko et al. 2004 J. procumbens

Fig. 2 continued

D (13) (Lin et al. 1995), and phyllamyricin E (14) (Lin et al. 1995) have been reported.

The second structural subclass includes C7-oxygenated Type I arylnaphthalene lactones. To date, 23 congeners have been isolated, in which the C7 of arylnaphthalene lactone is substituted with either the hydroxyl or methoxy group. Diphyllin (18) (Burden et al. 1969; Chen et al. 1996; Rao et al. 2006; Gözler et al. 1984; Anjaneyulu et al. 1981; Hesse et al. 1992; Susplugas et al. 2005; Sastry and Rao 1983), 
<smiles>COc1cc2c(O[C@@H]3O[C@H](C)[C@@H](O[C@H]4O[C@H](CO)[C@@H](O)[C@H](O)[C@H]4O)[C@H](C(C)=O)[C@H]3O)c3c(c(-c4ccc5c(c4)OCO5)c2cc1OC)C(=O)OC3</smiles>

65. Justiprocumin A

Zhang, Rumschlag-Booms et al. 2017

J. gendarussa<smiles>COC1=Cc2c(OC3O[C@H](COC4OC[C@@H](O)[C@H](O)[C@H]4O)[C@@H](O)[C@H](OC(C)=O)[C@H]3O)c3c(c(-c4ccc5c(c4)OCO5)c2c2cc(OC)c(OC)cc32)C(=O)OC1</smiles>

69. Mananthoside B Chen, Liu et al. 2002 M. patentiflora

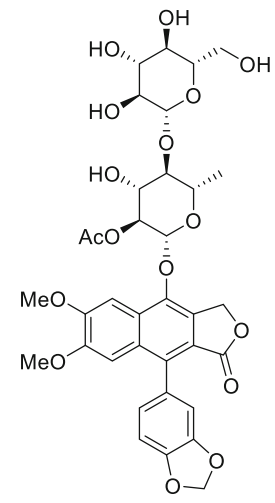

66. Justiprocumin B Zhang, Rumschlag-Booms et al. 2017

J. gendarussa<smiles>COc1cc2c(O[C@@H]3OC[C@@H](O[C@@H]4O[C@H](CO)[C@@H](O)[C@H](O)[C@H]4O)[C@H](OC)[C@H]3OC)c3c(c(-c4ccc5c(c4)OCO5)c2cc1OC)C(=O)OC3</smiles>

67. Ramontoside

Satyanarayana, Krupadanam et al. 1991

F. ramontchi<smiles>COc1cc2c(O[C@@H]3O[C@H](C)[C@@H](O[C@H]4OC(CO)[C@@H](O)[C@H](O)[C@H]4O)[C@H](O)[C@H]3O)c3c(c(-c4ccc5c(c4)OCO5)c2cc1OC)C(=O)OC3</smiles>

71. Mananthoside $C$ Tian, Hao et al. 2006 M. patentiflora<smiles>COc1cc2c(O[C@@H]3O[C@H](CO)[C@@H](O[C@H]4OC[C@H](O)[C@H](OC)[C@H]4OC)[C@H](O)[C@H]3O)c3c(c(-c4ccc5c(c4)OCO5)c2cc1OC)C(=O)OC3</smiles>

68. Cleistanthin C Anjaneyulu, Ramaiah et al. 1981

C. collinus<smiles>COc1cc2c(-c3ccc4c(c3)OCO4)c3c(c(OC4OC[C@](O)(CO)[C@H]4O[C@H]4OC[C@](O)(CO)[C@H]4O)c2cc1O)COC3=O</smiles>

72. Mananthoside $F$ Tian, Hao et al. 2006 M. patentiflora<smiles>COc1=c-c2c(OC3OC(COC4OC[C@@H](O)[C@H](O)[C@H]4O)[C@@H](O)[C@H](O)[C@H]3O)c3c(c(-c4ccc5c(c4)OCO5)c2c2cc(OC)c(OC)cc32)-c(=O)-o-1</smiles>

73. Mananthoside I Tian, $\mathrm{He}$ et al. 2008 M. patentiflora<smiles>COc1cc2c(OC3OC[C@@H](OC)[C@H](OC)[C@H]3O[C@H]3O[C@H](COC(C)=O)[C@@H](O)[C@H](O)[C@H]3O)c3c(c(-c4ccc5c(c4)OCO5)c2cc1OC)C(=O)OC3</smiles>

74. Taxodiifoloside Tuchinda et al., 2006 P. taxodiifolius

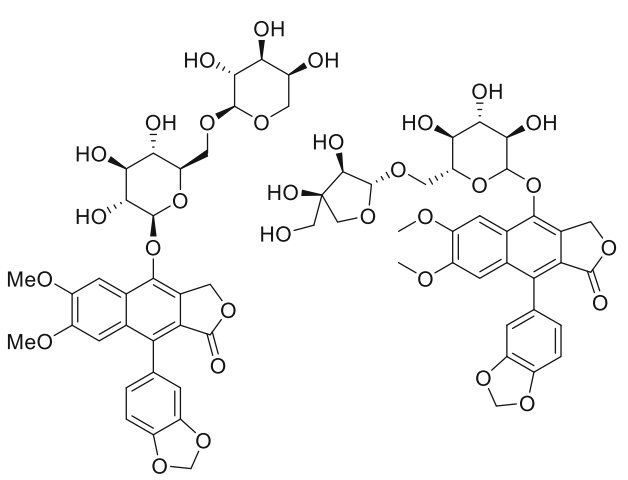

75. Arabelline Al-Abed, Sabri et al. 1990 H. buxbaumii Innocenti, Puricelli et al. 2002 H. patavinum
76. 7-O- $\beta$-D-apiofuranosyl-

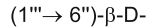
glucopyranosyldiphyllin Pandey et al. 2011 J. prostrata

Fig. 2 continued 
<smiles>COc1cc(C(OC2OC(CO)[C@H](O)[C@H](O)[C@H]2O)C(CO)Oc2c3c(c(-c4ccc5c(c4)OCO5)c4cc(OC)c(OC)cc24)C(=O)OC3)ccc1O</smiles>

77. Procumbenoside $M$ Jin et al. 2017

J. procumbens<smiles>COc1cc2c(OC3OCC4(COC(C)(C)O4)C3O)c3c(c(-c4ccc5c(c4)OCO5)c2cc1OC)C(=O)O3</smiles>

80. Pronaphthalide J Jin et al. 2014 J. procumbens<smiles>COc1cc2c(O[C@@H]3O[C@H](CO[C@H]4OC[C@@H](O)[C@H](O)[C@H]4O)[C@@H](OC(C)=O)[C@H](O[C@H]4O[C@H](CO)[C@@H](O)[C@H](O)[C@H]4O)[C@H]3OC(C)=O)c3c(c(-c4ccc5c(c4)OCO5)c2cc1OC)C(=O)OC3</smiles>

83. Mananthoside D Tian et al. 2006 M. patentiflora<smiles>COc1cc2c(OC3O[C@H](CO[C@H]4OC(CO)[C@@H](O)[C@H]4O)[C@@H](O)[C@H](O)[C@H]3O)c3c(c(-c4ccc5c(c4)OCO5)c2cc1OC)C(=O)OC3</smiles>

78. Reticulatuside A Ma et al. 2012

P. reticulatus<smiles>COc1cc2c(OC)cc3c(O[C@@H]4OC[C@@H](O)[C@H](O[C@@H]5OC[C@H](O)[C@H](O)[C@H]5O)[C@H]4O)c4c(c(-c5ccc6c(c5)OCO6)c3c2cc1OC)C(=O)OC4</smiles>

81. Diphyllin 7-O- $\alpha$-L-arabinopyranosyl-(1"' $\rightarrow 3$ ")- $\alpha-L-$ arabinopyranoside Yu et al. 2016

$P$. glaucus

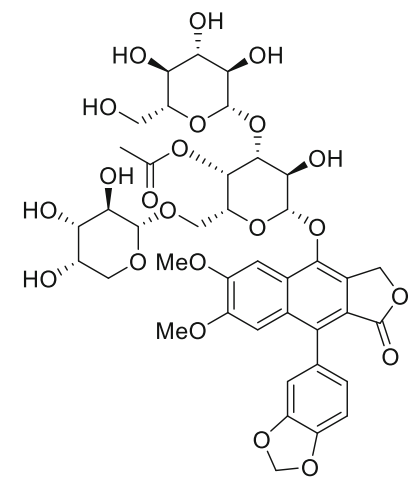

84. Mananthoside $E$ Tian et al. 2006 M. patentiflora<smiles>COc1cc2c(-c3ccc4c(c3)OCO4)c3c(c(OC4OC[C@H](O)[C@H](O)[C@H]4O)c2cc1OC1O[C@H](CO)[C@@H](O)[C@H](O)[C@H]1O)COC3=O</smiles>

79. Reticulatuside B Ma et al. 2012

$P$. reticulatus<smiles>COc1cc2c(O[C@@H]3OC[C@@](O)(COC4OC[C@@H](O)[C@H](O[C@H]5O[C@H](CO)[C@@H](O)[C@H](O)[C@H]5O)[C@H]4O)C3OC(C)=O)c3c(c(-c4ccc5c(c4)OCO5)c2cc1OC)C(=O)OC3</smiles>

82. Qudsine Al-Abed, Sabri et al. 1990 H. buxbaumii
85. Procumbenoside E Wu et al. 2012 J. procumbens

Fig. 2 continued 
<smiles>COc1cc2c(O[C@@H]3OC[C@@H](OC)[C@H](OC)[C@H]3O[C@@H]3O[C@H](CO)[C@@H](O)[C@H](O)[C@H]3OC)c3c(c(-c4ccc5c(c4)OCO5)c2cc1OC)C(=O)OC3</smiles>

86. Cleistanthoside $A$ Zhang, Ma et al. 2014

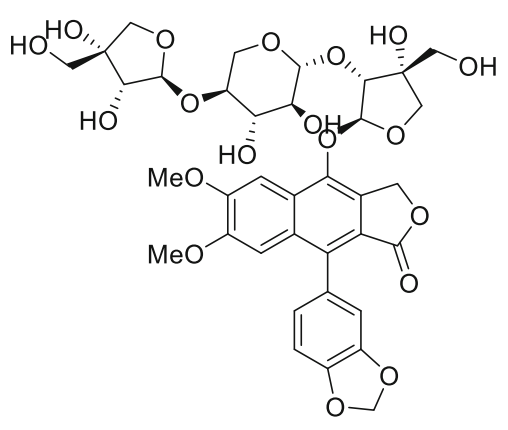

87. Patavine Innocenti, Puricelli et al. 2002 H. patavinum

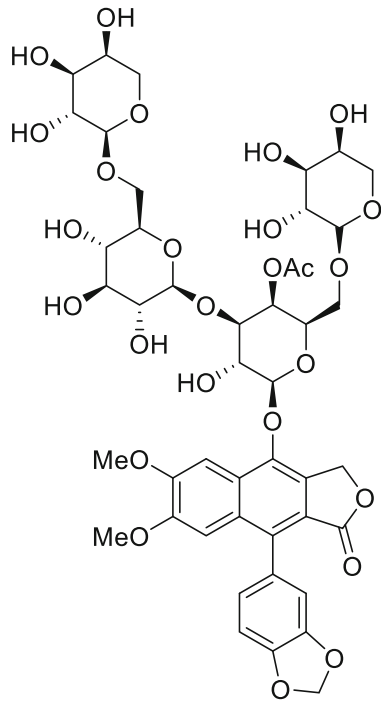

88. Mananthoside $\mathrm{J}$ Tian, He et al. 2008 M. patentiflora<smiles>COc1cc2c(O[C@@H]3OC[C@@](O)(CO[C@H]4OC[C@@H](O)[C@H](O)[C@H]4O)[C@H]3O[C@H]3OC[C@@H](O)[C@H](O[C@@H]4OC[C@](O)(CO)[C@H]4O)[C@H]3O)c3c(c(-c4ccc5c(c4)OCO5)c2cc1OC)C(=O)OC3</smiles>

89. Ciliatoside B Day et al. 2000 J. ciliata<smiles>COc1cc2c(O[C@@H]3OC[C@](O)(CO[C@H]4OC[C@@H](O)[C@H](O)[C@H]4O)[C@H]3O[C@@H]3OC[C@@H](O)[C@H](O)[C@H]3O)c3c(c(-c4ccc5c(c4)OCO5)c2cc1OC)C(=O)OC3</smiles>

90. Ciliatoside $A$

Burden, Crombie et al. 1969

J. ciliata

Fig. 2 continued

chinensinaphthol (19) (Chen et al. 1996; Day et al. 1999Ghosal et al. 1974), taiwanin E (20) (Chen et al. 1996; Anjaneyulu et al. 1981; Wang et al. 2014), cleistanone (21) (Ramesh et al. 2003), 6'-hydroxyjusticidin A (27) (Yang et al. 2006), 5-hydroxyjusticidin A (36) (Tian et al. 2006a, b), dehydropodophyllotoxin (24) (Novelo et al. 1993), 2'-hydroxyjustirumalin (25) (Rezanka et al. 2009), justicidin A (26) (Burden et al. 1969; Day et al. 2002; Wu and Wu 2006; Lin et al. 1995; Hesse et al. 1992; Day et al. 1999; Susplugas et al. 2005), Khalid et al. 1981, haplomyrtin (22) (Wu and Wu 2006; Evcim et al. 1986), 5-methoxydehydropodophyllotoxin (23) (Novelo et al. 1993), cilinaphthalide A (28) (Day et al. 1999), cilinaphthalide B (29) (Weng et al. 2004; Day et al. 1999), chinensinaphthol methyl ether (30) (Luo et al. 2014), phyllanthusmin A (31) (Wu and Wu 2006; Ren et al. 2014), justicidin F (32) (Chen et al. 1996; Day et al. 1999), justicidin P (33) (Wang and Ripka 1983), justicinol (34) (Susplugas et al. 2005), and justicidinoside (35) 


\section{Type 2}

\section{A. Type II arylnaphthalene lactones}<smiles>COc1cc2cc3c(c(-c4ccc5c(c4)OCO5)c2cc1OC)COC3=O</smiles>

91. Retrojusticidin $B$ Lin, Lee et al.1995

P. myrtifolius<smiles>COc1ccc(-c2c3c(cc4cc5c(cc24)OCO5)C(=O)OC3)cc1OC</smiles>

95. Retrochinensin Ghosal and Banerjee 1979 J. prostata<smiles>O=C1OCc2c1cc1ccc(O)c(OC3O[C@H](CO)[C@@H](O)[C@H](O)[C@H]3O)c1c2-c1ccc2c(c1)OCO2</smiles>

99. Elenoside Navarro, Alonso et al. 2001 J. hyssopifolia<smiles>COc1cc2cc3c(c(-c4cc5c(cc4OC)OCO5)c2cc1OC)COC3=O</smiles>

92. Procumphthalide $A$ Weng, Ko et al. 2004

J. procumbens<smiles></smiles>

96. Justicidin E Wada and Munakata 1970 J. procumbens<smiles>COc1cc2c(OC)c3cc4c(c(-c5ccc6c(c5)OCO6)c3cc2c(OC)c1OC)C(=O)OC4</smiles>

93. Phyllamyricin A Windayani 2014 P. myrtifolius<smiles>O=C1OC(O)c2c1cc1ccc3c(c1c2-c1ccc2c(c1)OCO2)OCO3</smiles>

97. Jusmicranthin Rajasekhar and Subbaraju 2000 J. neesii<smiles>COc1cc2cc3c(c(-c4ccc5c(c4)OCO5)c2c(OC)c1OC)COC3=O</smiles>

101. Chaihunaphthone Liu et al 2008 B. marginatum<smiles>COc1cc(-c2c3c(cc4cc(O)c(O)cc24)C(=O)OC3)ccc1O</smiles>

94. Detetrahydroconidendrin Kuo et al. 1990

J. formosana<smiles>O=C1OCc2c1cc1ccc3c(c1c2-c1ccc2c(c1)OCO2)OCO3</smiles>

98. Helioxanthin Ban, Lee et al. 2002 A. chiisanensis Ghosal, Chauhan et al. 1974 $P$. chinensis Burden, Crombie et al. 1969 H. scabra<smiles>CCOC1OC(=O)c2cc3cc(OC)c(O)cc3c(-c3ccc(O)c(OC)c3)c21</smiles>

100. Vitexdoin I

Zheng et al. 2014 $\checkmark$. negundo<smiles>COc1ccc(-c2c3c(cc4cc(O)c(OC)cc24)C(=O)OC3)cc1O</smiles>

102. $5,3^{\prime}$-dihydroxy-4,4'dimethoxy-2,7'-cycloligna7,7'-diene-9,9'-lactone Zhang et al. 2010 L. elegans

Fig. 3 Structure and isolations of Type II arylnaphthalene lactones

(Asano et al. 1996), justalakonin (37) (Kavitha et al. 2003), procumbenoside K (38) (Jin et al. 2017), pronapthalide A (39) (Jin et al. 2014) and 4'-Odemthyl-7-O-methyldehydropodophylotoxin (Wei at al. 2018) have been reported. Among these, justicidin $\mathrm{P}$ is a 7-oxygenated derivative of justicidin A and justicidinoside B is the glycosylated product of 6'-hydroxyjusticidin A.
The third subclass of Type I arylnaphthalene lactones are 7-O-glycosyl congeners. A variety of saccharides are conjugated at the 7-hydroxy group of diphyllin, haplomyrtin, taiwanin E, and 4-hydroxydaurinol. The 7-O-glycosylated Type I naphthalene lactones presented in Fig. 1 summarize the naturally occurring glycosylated congeners. Monosaccharideconjugated derivatives include tuberculatin (41) 


\section{B. 7- Oxygenated Type II arylnaphthalene lactones}<smiles>COc1cc2c(OC)c3c(c(-c4ccc5c(c4)OCO5)c2cc1OC)COC3=O</smiles>

103. Justicidin C (Neojusticidin B)

Day, Chiu et al. 1999

J. ciliata

Ohta and Munakata

1970

J. procumbens<smiles>COc1cc2c(-c3ccc4c(c3)OCO4)c3c(c(OC)c2cc1O)C(=O)OC3</smiles>

107. Neojusticin C

Yang, Wu et al. 2006

J. procumbens<smiles>COc1cc2c(OC)c3c(c(-c4cc5c(cc4O)OCO5)c2cc1OC)C(=O)OC3</smiles>

104. 6'-Hydroxyjusticidin C Yang, Wu et al. 2006 J. procumbens<smiles>COc1c2c(c(-c3ccc4c(c3)OCO4)c3cc4c(cc13)OCO4)CC(=O)C2</smiles>

105. Justicidin D (Neojusticidin A) Ohta and Munakata 1970 J. procumbens<smiles>COc1cc2c(OC)c3c(c(-c4cc5c(cc4OC4O[C@H](CO)[C@@H](O)[C@H](O)[C@H]4O)OCO5)c2cc1OC)COC3=O</smiles>

106. Justicidinoside $A$

Asano, Chiba et al. 1996

J. procumbens

Fig. 3 continued

(diphyllin apioside) (Susplugas et al. 2005; Innocenti et al. 2002), cleistanthin D (42) (Anjaneyulu et al. 1981), diphyllin acetylapioside (43) (Nukul et al. 1987; Prieto et al. 2002), haplomyrtoside (44) (Gözler et al. 1996), cleistanthin A (45) (Anjaneyulu et al. 1981; Sastry and Rao 1983; Tuchinda et al. 2006), cleistanthin A methyl ether (46) (Tuchinda et al. 2006), cleistanthin B (47) (diphyllin O-glycoside) (Anjaneyulu et al. 1981; Al-Abed et al. 1990; Ren et al. 2014), mananthoside A (48) (Chen et al. 2002), phyllanthusmin B (49) (Lin et al. 1995; Ren et al. 2014), phyllanthusmin C (50) (Lin et al. 1995; Ren et al. 2014), phyllanthusmin D (51) (Ren et al. 2014), phyllanthusmin E (52) (Ren et al. 2014), procumbenoside C (53) (Liu et al. 2008a), procumbenoside D (54), Liu et al. (2008b), patentiflorin A (55) (Susplugas et al. 2005), patentiflorin B (56) (Susplugas et al. 2005), procumbenoside I (57) (Jin et al. 2017) acutissimalignan A (58) (Tuchinda et al. 2008) 7-O$\beta$-D-glucopyranosyljusticidin B (59) (Borges et al. 2018), 7-O-( $\beta$-D-glucopyranosyl)-dehydropodophyllotoxin (60) (Liu et al. 2015) and 4"-O-acetylpatentiflorin B (61) (Susplugas et al. 2005). Disaccharideconjugated congeners include majidine (62) (Al-Abed et al. 1990; Innocenti et al. 2002), procumbenoside A (63) (Day et al. 2002), procumbenoside B (64) (Weng et al. 2004), justiprocumin A (65) (Zhang et al. 2017), justiprocumin B (66) (Zhang et al. 2017), ramontoside (67) (Satyanarayana et al. 1991), cleistanthin C (68) (Anjaneyulu et al. 1981), mananthoside B (69) (Chen et al. 2002), 4"-O-acetylmananthoside B (70) (Susplugas et al. 2005), mananthoside C (71) (Tian et al. 2006a, b), mananthoside F (72) (Tian et al. 2006a, 2006b), mananthoside I (73) (Tian et al. 2008), taxodiifoloside (74) (Tuchinda et al. 2006), arabelline (75) (Al-Abed et al. 1990; Innocenti et al. 
2002), and 7-O- $\beta$-D-apiofuranosyl-(1" $\rightarrow 6 ")-\beta-D-$ glucopyranosyldiphyllin (76) (Pandey et al. 2011), procumbenoside M (77) (Jin et al 2017), reticulatuside A (78) (Ma et al. 2012), reticulatuside B (79) (Ma et al. 2012), pronapthalide J (80) (Jin et al. 2014), Diphyllin $7-\mathrm{O}-\alpha$-L-arabinopyranosyl-( 1 " $\rightarrow 3$ ")- $\alpha$-L-ara-

binopyranoside (81) ( $\mathrm{Yu}$ et al. 2016) and cleistanthoside A (86) (Zhang et al. 2014). Eight trisaccharideconjugated diphyllins, namely mananthoside D (83) (Tian et al. 2006a, b), mananthoside E (84) (Tian et al. 2006a, b), procumbenoside E (85) (Wu et al. 2012), mananthoside J (88) (Tian et al. 2006a, b), patavine (87) (Innocenti et al. 2002), ciliatoside B (89) (Day et al. 2000), ciliatoside A (90) (Burden et al. 1969), and qudsine (82) (Al-Abed et al. 1990), have been reported.

Type II arylnaphthalene lactones are characterized by the trans relationship of lactone carbonyl and the aryl group. Twelve Type II congeners were isolated and structurally elucidated, including retrojusticidin B (91) (Lin et al. 1995), procumphthalide A (92) (Weng et al. 2004), phyllamyricin A (93) (Windayani et al. 2014), detetrahydroconidendrin (94) (Kuo et al. 1990), retrochinensin (95) (Ghosal and Banerjee 1979), justicidin E (96) (Wada and Munakata 1970), jusmicranthin (97) (Rajasekhar and Subbaraju 2000), helioxanthin (98) (Ban et al. 2002; Ghosal et al. 1974; Burden et al. 1969), and elenoside (99) (Navarro et al. 2001), vitexdoin I (101) (Zheng et al. 2014), Chaihunaphthone (102) (Liu et al. 2008a, b) and 5,3'dihydroxy-4,4' -dimethoxy-2,7' -cycloligna-7,7'-diene-9,9'-lactone (103) (Zhang et al, 2010)

\section{Pharmacological activities}

Cytotoxic activities

The reported antiproliferative activities of natural arylnaphthalene lactones are presented in Fig. 4. Significant cytotoxic activity was observed with justicidin A (26) and tuberculatin (41) against human hepatoma cellular carcinoma (Hep3B and HepG2), human breast cancer (MCF-7 and MCF-7-ras), human cervical carcinoma ( $\mathrm{SiHa}$ ), and other cancer cell lines. In addition, these two compounds strongly enhanced tumor-necrosis factor $\alpha$ (TNF- $\alpha$ ) generation in lipopolysaccharide (LPS)-stimulated RAW 264.7 cells (Day et al. 2002). Later, 6'-hydroxyjusticidin A
(27), which was isolated from Justicia procumbens, was evaluated for its cytotoxicity against human cancer cell lines. It showed remarkable inhibitory activity in human bladder cancer cells (EJ) with 50\% inhibitory concentration $\left(\mathrm{IC}_{50}\right.$ ) values of $57.1 \mu \mathrm{M}$ and enhanced the generation of reactive oxygen species and induced apoptosis through the caspase pathway (He et al. 2012). Similar results of the mechanism of action were reported by Luo and $\mathrm{Hu}$ et al. in 2014. They isolated five lignans, $6^{\prime}$-hydroxyjusticidin A (27), justicidin H (3), justicidin B (1), chinensinaphthol methyl ether (30), and taiwanin E methyl ether (32) from J. procumbens and tested their cytotoxic activities. Justicidin $\mathrm{H}(\mathbf{3})$ exhibited the best inhibitory activity against human promyelocytic leukemia (HL60) and mouse lymphocytic leukemia (L1210 and P3881D1) cells with an $\mathrm{IC}_{50}$ ranging from 3.9 to $26.2 \mu \mathrm{M}$ (Luo et al. 2014). To investigate the mechanism of action of justicidin $\mathrm{H}$ (3), these authors also evaluated its effects on human leukemia K562 cells. The $\mathrm{IC}_{50}$ of justicidin $\mathrm{H} \mathrm{(3)}$ was $15.07 \mu \mathrm{M}$ for $\mathrm{K} 562$ cells and reduced mitochondria membrane potential (deltapsi(m)). It also increased the expression of TRPC6 related to regulating calcium homeostasis in cell signaling and induced apoptosis through the caspase pathway (Luo et al. 2018).

Diphyllin (18) was tested to investigate whether it could act as a vacuolar-ATPase (V-ATPase) inhibitor against human gastric cancer cells (SGC7901) and esophageal cancer cells (TE-1 and ECA-109). The $\mathrm{IC}_{50}$ for SGC7901 was demonstrated to be $7.8 \mu \mathrm{M}$. Diphyllin (18) also inhibited the expression of $\mathrm{V}$-ATPases in a dose-dependent manner. In addition, the transmembrane $\mathrm{pH}$ gradient was reversed, thereby causing tumor microenvironment acidification (Shen et al. 2011). It also showed significant inhibition against TE-1 and ECA-109 cells with $\mathrm{IC}_{50}$ values of 0.3 and $0.2 \mu \mathrm{M}$, respectively, with S-phase arrest and reduced V-ATPase activity. Reportedly, diphyllin inhibited mammalian target of rapamycin complex 1 (mTORC1), hypoxia-inducible factor-1 $\alpha$ (HIF-1 $\alpha$ ), and vascular endothelial growth factor (VEGF) mRNA expression (Chen et al. 2018). Three diphyllin glycosides cleistanthin A (45), cleistanthoside A (86), and cleistanthoside A tetraacetate were also evaluated for their effect as V-ATPases and their cytotoxicity against human cell lines. Apart from cleistanthoside A (86), cleistanthin A (45) and cleistanthoside A tetraacetate were more potent than paclitaxel against 
<smiles>COc1cc2c(OC)c3c(c(-c4ccc5c(c4)OCO5)c2cc1OC)C(=O)OC3</smiles>

26 Justicidin A<smiles>COc1cc2c(OC)c3c(c(-c4cc5c(cc4O)OCO5)c2cc1OC)C(=O)OC3</smiles>

27 6'-Hydroxyjusticidin A

$\mathrm{IC}_{50}[\mathrm{\mu g} / \mathrm{ml}]$

1.81 HCT-8 0.49 BCG-823

3.61 BEL-7402 $0.37 \quad$ KB

$\begin{array}{llll}2.22 & \text { KETR3 } & 3.49 & \text { MCF-7 }\end{array}$

0.16 HELA

(Wang, Tong et al. 2014)<smiles>COc1cc2c(O)c3c(c(-c4ccc5c(c4)OCO5)c2cc1OC)C(=O)OC3</smiles>

18 Diphyllin

$\mathrm{ED}_{50}[\mathrm{\mu g} / \mathrm{ml}]$

3.6 Нер3B 2.97 SGC7901

0.4 HepG2 0.106 ECA-109

$\begin{array}{lll}2.7 & 212 & 0.078 \mathrm{TE}-1\end{array}$

(Day, Lin et al. 2002,

Shen, Zou et al. 2011

Chen, Liu et al. 2011)

(Day, Lin et al. 2002, Dan et al. 2010

Weng, Ko et al. 2004)<smiles>COc1cc2cc3c(c(-c4ccc5c(c4)OCO5)c2cc1OC)C(=O)OC3</smiles>

1 Justicidin B

$\mathrm{IC}_{50}[\mu \mathrm{g} / \mathrm{ml}]$

$0.2 \quad \mathrm{~KB}$

3.3 PBM

3.2 Jurkat $\mathrm{T}$

(Burden, Crombie et al. 1969

Gertsch, Tobler et al. 2003)

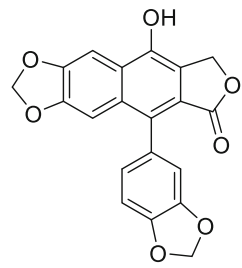

20 Taiwanin E

$\mathrm{IC}_{50}[\mu \mathrm{g} / \mathrm{ml}]$

$0.54 \quad$ MCF-7

(Wang, Tseng et al. 2014)

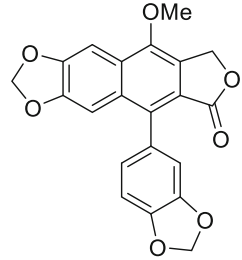

32 Justicidin $F$ (Taiwanin E methyl ether) $\mathrm{ED}_{50}[\mathrm{\mu g} / \mathrm{ml}]$

2.6 HT-3

(Day, Chiu et al. 1999)<smiles>COc1cc(-c2c3c(c(OC)c4cc(OC)c(OC)cc24)COC3=O)ccc1O</smiles>

28 Cilinaphthalide A $\mathrm{ED}_{50}[\mu \mathrm{g} / \mathrm{ml}]$

$1.3 \mathrm{~T}-24$

3 CaSki

$0.3 \quad \mathrm{HT}-3$

(Day, Chiu et al. 1999)<smiles>COc1cc2c(-c3ccc4c(c3)OCO4)c3c(cc2cc1O)COC3=O</smiles>

4 Daurinol

$\mathrm{IC}_{50}[\mu \mathrm{g} / \mathrm{ml}]$

0.71 HCT116

(Kang, Oh et al. 2011)<smiles>COc1cc(-c2c3c(cc4cc5c(cc24)OCO5)COC3=O)cc2c1OCO2</smiles>

6 Koelreuterin-1

$\mathrm{ED}_{50}[\mu \mathrm{g} / \mathrm{ml}]$

0.0035 SK-MEL-5

0.0067 SK-OV-3

(Song, Zhang et al. 1994) (Burden, Crombie et al. 1969)

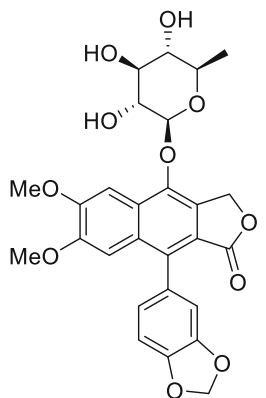

55 Patentiflorin A

$\mathrm{IC}_{50}[\mathrm{\mu g} / \mathrm{ml}]$

$2.24 \quad \mathrm{~KB}$

(Wu and Wu 2006)<smiles>COc1cc2cc3c(c(-c4cc5c(cc4O)OCO5)c2cc1OC)C(=O)OC3</smiles>

3 Justicidin $\mathrm{H}$

(6'-Hydroxyjusticidin B)

$\mathrm{IC}_{50}[\mu \mathrm{g} / \mathrm{ml}]$

$7.61 \quad \mathrm{~K} 562$<smiles>COc1cc2c(O[C@@H]3OC[C@](O)(CO)[C@H]3O[C@H]3OC[C@@H](O)[C@H](O)[C@H]3O)c3c(c(-c4ccc5c(c4)OCO5)c2cc1OC)C(=O)OC3</smiles>

63 Procumbenoside A $\operatorname{ED} 50[\mu \mathrm{g} / \mathrm{ml}]$

3.1 Hep3B

3.9 HepG2

3.1212

(Day, Lin et al. 2002)
$0.002 \quad \mathrm{~KB}$

$0.0015 \quad$ MCF-7

(Susplugas, Hung et al. 2005)
Fig. 4 Arylnaphthalene lactones with cytotoxic activity against different cell lines. Hep3B, human cervical carcinoma; HepG2, human hepatoma cell; MCF-7, human breast cancer cell; MCF-7 ras, Ha-ras oncogene transformed from MCF-7; EJ, human bladder cell; K562, human leukemia cell; SGC7901, human

HepG 2 cells with the $\mathrm{IC}_{50}$ values of 36 and $39 \mathrm{nM}$, respectively. They also inhibited V-ATPase activity, which is critical to tumor invasion and metastasis gastric cancer cell; TE-1 and ECA-109, human esophageal cancer cells; SK-OV-3, human ovarian carcinoma; SK-MEL-5, melanoma; PLC/PRF/5, human hepatoma; HT-3, SiHa, and CaSki, human cervical carcinoma

development. At nanomolar concentrations, they neutralize the $\mathrm{pH}$ of lysosomes (Zhang et al. 2014).

A bioassay-guided fractionation of the stems and roots of Phyllanthus oligospermus resulted in the 
isolation of three arylnaphthalene lignan lactones, phyllanthusmin A-C (31, 49, 50). The most active compound was phyllanthusmin A (31) showing a marked cytotoxic effect against mouse leukemia (P388 ) and human epidermoid carcinoma (KB) cells with $\mathrm{IC}_{50}$ values of 0.13 and $2.24 \mu \mathrm{g} / \mathrm{mL}$, respectively (Wu and Wu 2006). Phyllanthusmin A-E (31, 49-52), diphyllin (18), and cleistanthin B (47) were also evaluated for cytotoxicity against colon cancer cells (HT-29). Phyllanthusmin D (51) was the most potent with $\mathrm{IC}_{50}$ values at $170 \mathrm{nM}$; however, cleistanthin $\mathrm{B}$ (47) and phyllanthusmin A (31) were inactive. These results suggest that the presence of more lipophilic acetyl groups results in higher cytotoxicity. In this connection, mechanistic studies of phyllanthusmin D (51) were also evaluated. It was found that unlike etoposide, phyllanthusmin D (51) did not mediate its cytotoxic effects by inhibiting DNA topoisomerase II $\alpha$ but did so by inducing HT-29 apoptosis through caspase-3 activation (Ren et al. 2014). However, daurinol (4) acts as a catalytic human topoisomerase II $\alpha$ inhibitor and demonstrated significant cytotoxic activity against human colorectal cancer cells (HCT116) with an $\mathrm{IC}_{50}$ of $2.03 \mu \mathrm{M}$. It induced S-phase arrest through the increased expression of cyclin E and A (Kang et al. 2011). In a further investigation, Woo et al. (2017) evaluated daurinol (4) for anti-metastatic activity against human breast cancer cells (MDA-MB-231) and human lung cancer cells (A549). Daurinol (3) decreased the expression of focal adhesion kinase, which is hyper-activated and overexpressed in most solid tumors, but did not block the AKT pathway in both cell lines. Using a trans-well assay, daurinol (3) was found to inhibit migration and invasion (Woo et al. 2017).

Among the eight compounds, cilinaphthalide A (28), cilinaphthalide B (29), chinensinaphthol methyl ether (30), justicidin A (26), neojusticin B (103), taiwanin E methyl ether (32), chinensinaphthol (19), and diphyllin (18) were isolated from the whole plant of Justicia ciliate. The potent cytotoxic effects of justicidin A (26) were reported against human cervical carcinoma (CaSki, SiHa, and HT-3) and human hepatoma (PLC/PRF/5 and T-24) cells with $\mathrm{IC}_{50}$ values at $3.0 \times 10^{-3}, 7.4 \times 10^{-3}, 1.8 \times 10^{-3}$, $2.2 \times 10^{-3}$, and $2.0 \times 10^{-3} \mu \mathrm{g} / \mathrm{mL}$, respectively (Day et al. 1999). Significant cytotoxicity was observed for most of the compounds, justicinol (34), patentiflorin A-B $(\mathbf{5 5}, \mathbf{5 6}), 4^{\prime \prime}$ - $O$-acetylpatentiflorin B
(61), and $4^{\prime \prime}-O$-acetylmananthoside B (70), isolated from the leaves and stems of Justicia patentiflora with nanomolar values of $\mathrm{IC}_{50}$. The most active compound was patentiflorin A (55) with the nanomolar range of $\mathrm{IC}_{50} 0.004$ and 0.003 against mouth epidermoid carcinoma (KB) and breast cancer (MCF-7) cells, respectively (Susplugas et al. 2005).

Antiplatelet aggregation activities

In 1996, Chen et al. determined the 50\% inhibitory activity to the arachidonic acid (AA)-induced aggregation of rabbit platelets at $20 \mu \mathrm{g} / \mathrm{mL}$ from the EtOH extract of the whole plant of $J$. procumbens. They isolated nine arylnaphthalide lignans, neojusticin A (105), justicidin B (1), justicidin A (26), taiwanin E methyl ether (32), neojusticin B (103), chinensinaphthol methyl ether (30), taiwanin E (20), chinensinaphthol (19), and diphyllin (18), from $J$. procumbens and evaluated these for their antiplatelet activity. All compounds were less effective than indomethacin; however, neojusticin A (105), taiwanin E methyl ether (32), justicidin B (1), and taiwanin E (20) were more active than aspirin with $\mathrm{IC}_{50}$ values at 1.1, 1.7, 8.0, and $8.0 \mu \mathrm{M}$, respectively (C.-C. Chen et al. 1996). In a further study, Weng et al. isolated two additional new arylnaphthalide lignans, procumbenoside B (64) and cilinaphthalide B (29) from $J$. procumbens and tested the antiplatelet effects induced by adrenaline in human platelet-rich plasma. Cilinaphthalide B (29), justicidin A (26), and taiwanin E methyl ether (32) exhibited a moderate antiplatelet activity in a concentrationdependent manner. Among these, at high concentrations, taiwanin E methyl ether (32) completely abolished the aggregation with an $\mathrm{IC}_{50}$ value of $27.7 \mu \mathrm{M}$ and inhibited the secondary phase aggregation at low concentrations. These results indicate that justicidin A (26) and taiwanin E methyl ether (32) likely suppress cyclooxygenase activity and reduce thromboxane formation (Weng et al. 2004) (Fig. 5).

\section{Antiviral activities}

A series of lignans isolated from $J$. procumbens were tested for activities against the vesicular stomatitis virus. Justicidin A-B (26, 1), diphyllin (18), diphyllin apioside (41), and diphyllin apioside-5-acetate exhibited strong antiviral activities. Their minimum inhibitory concentration (MIC) values were less than 
<smiles>COc1cc2cc3c(c(-c4ccc5c(c4)OCO5)c2cc1OC)C(=O)OC3</smiles>

1 Justicidin B $\mathrm{IC}_{50}(\mu \mathrm{M})$

$8.0 \pm 1.2$<smiles>O=C1OCc2c1c(-c1ccc3c(c1)OCO3)c1cc3c(cc1c2O)OCO3</smiles>

20 Taiwanin E $\mathrm{IC}_{50}(\mu \mathrm{M})$

$8.0 \pm 1.1$

Fig. 5 Arylnaphthalene lactones with antiplatelet activity. Inhibitory concentrations (IC) were determined in arachidonic acid (AA)-induced aggregation of rabbit platelets (C.-C. Chen

$0.25 \mu \mathrm{g} / \mathrm{mL}$ whereas $6^{\prime}$-glucosides justicidinoside A-C (106, 35, 9) and Type II justicidin C and D $(\mathbf{1 0 3}, \mathbf{1 0 5})$ exhibited lower antiviral activity (the MICs ranged from 16 to $125 \mu \mathrm{g} / \mathrm{mL}$ ). It is tempting to suggest that the weak activity of justicidinoside A-C $(\mathbf{1 0 6}, \mathbf{3 5}, \mathbf{9})$ is because of the steric bulk of their sugar moiety and that Type I arylnaphthalene lactones were more effective than Type II (Luo et al. 2018). Using the standard plaque reduction assay against human cytomegalovirus, only taiwanin C (2) and retrojusticidin $\mathrm{B}(\mathbf{9 1})$ showed clear antiviral activity with half maximal effective concentration $\left(\mathrm{EC}_{50}\right)$ values at 1.2 and $7.2 \mu \mathrm{M}$, respectively (Chen et al. 1996). Similar antiviral activities were reported for helioxanthin (98) in HepG2.2.15 cells using Southern blot hybridization with the $\mathrm{EC}_{50}$ value at $1 \mu \mathrm{M}$. Helioxanthin (98)<smiles>COc1c2c(c(-c3ccc4c(c3)OCO4)c3cc4c(cc13)OCO4)COC2=O</smiles>

105 Justicidin D

(Neojusticin A)

$\mathrm{IC}_{50}(\mu \mathrm{M})$

$1.1 \pm 0.3$<smiles>COc1c2c(c(-c3ccc4c(c3)OCO4)c3cc4c(cc13)OCO4)C(=O)OC2</smiles>

32 Justicidin F

(Taiwanin $\mathrm{E}$ methyl ether)

$\mathrm{IC}_{50}(\mu \mathrm{M})$

$1.7 \pm 0.3$

$$
27.6^{a}
$$

et al. 1996), a. Platelet aggregation induced by adrenaline in human platelet-rich plasma (Weng et al. 2004)

reduced $3.5 \mathrm{~kb}$ of hepatitis $\mathrm{B}$ virus mRNA in a dosedependent manner with $\mathrm{EC}_{50}$ values at $0.09 \mu \mathrm{M}(\mathrm{Li}$ et al. 2005). In addition, helioxanthin (98) also exhibited strong antiviral activities against hepatitis $\mathrm{C}$ virus and herpes simplex virus type 1 with $\mathrm{EC}_{50}$ values at 3 and $2 \mu \mathrm{M}$, respectively, but showed weak activity against herpes simplex virus type 2 and Epstein-Barr virus with $\mathrm{EC}_{50}$ values at 35 and above $20 \mu \mathrm{M}$, respectively (Yeo et al. 2005) (Table1).

\section{Anti-HIV activities}

Anti-HIV bioassays with six lignans, phyllamyricin A (93), phyllamyricin B, phyllamyricin C (12), retrojusticidin B (91), justicidin A (26), and justicidin B (1) isolated from Phyllanthus myrtifolius were first
Table 1 Arylnaphthalene lactones with antiviral activities

\begin{tabular}{lll}
\hline Compound & Virus & MIC $(\mu \mathrm{g} / \mathrm{mL})$ \\
\hline Justicidin A (26) & Vesicular stomatitis virus & 0.13 \\
Justicidin B (1) & Vesicular stomatitis virus & $\geq 0.06$ \\
Diphyllin (18) & Vesicular stomatitis virus & 0.25 \\
Tuberculatin (41) & Vesicular stomatitis virus & 0.25 \\
& & $\mathrm{EC}_{50}[\mu \mathrm{M}]$ \\
Taiwanin C (2) & Cytomegalovirus & 1.2 \\
Retrojusticidin B (91) & Cytomegalovirus & 7.2 \\
Helioxanthin (98) & Cytomegalovirus & 7.3 \\
& Hepatitis B virus & 1 \\
& Hepatitis C virus & 3 \\
\hline
\end{tabular}

MIC, minimum inhibitory concentration; $\mathrm{EC}_{50}$, half maximal effective concentration 
Table 2 Anti-HIV activities of arylnaphthalene lignan lactones

\begin{tabular}{lc}
\hline Compound & $\mathrm{IC}_{50}$ \\
\hline $\begin{array}{l}\text { Retrojusticidin B (91) }{ }^{\mathrm{a}} \\
\text { HIV-RT }\end{array}$ & $5.5 \mu \mathrm{M}$ \\
Justiprocumin B (66) & \\
BAL & $15 \mathrm{nM}$ \\
SF162 & $15 \mathrm{nM}$ \\
LAV0.04 & $14 \mathrm{nM}$ \\
89.6 & $21 \mathrm{nM}$ \\
\hline
\end{tabular}

BAL, SF162, LAV0.04, and 89.6 are HIV-1 clinical isolates

${ }^{a}$ Using reverse transcriptase assay

${ }^{\mathrm{b}}$ Using standardized human peripheral blood mononuclear cell culture assay (PBMC assay)

conducted by Chang et al. (1995) using human immunodeficiency virus-1 reverse transcriptase assay (HIV-RT). Among these, phyllamyricin B and retrojusticidin $\mathrm{B}$ (91) were shown to contribute to the selective inhibitory effect against HIV-RT with $\mathrm{IC}_{50}$ values at 3.5 and $5.5 \mu \mathrm{M}$, respectively, whereas they exhibited much lower activity against human DNA polymerase- $\alpha$ (hDNAP- $\alpha$ ) with $\mathrm{IC}_{50}$ values at 289 and $989 \mu \mathrm{M}$ (Chang et al. 1995) (Table 2). In a subsequent study in 1996, Lee et al. identified additional lignans from $P$. myrtifolius and evaluated their anti-HIV activities. Phyllamyricin $\mathrm{B}$ and $\mathrm{C}$ were inactive and phyllamyricin E (14) exhibited very low anti-HIV-RT activity; however, phyllamyricin A (93) showed an increase in HIV-RT activity by $65 \%$ at $1.89 \mu \mathrm{M}$ (Lee et al. 1996). From the stems and barks of Justicia gendarussa justiprocumin $\mathrm{A}$ and $\mathrm{B}(\mathbf{6 5}, \mathbf{6 6})$ were isolated and justiprocumin B (66) was assayed for its
anti-HIV activity against four HIV-1 isolates using a standardized human peripheral blood mononuclear cell culture assay. The HIV-1 isolates BAL, SF162, LAV0.04, and 89.6 were used. Justiprocumin B exhibited $\mathrm{IC}_{50}$ values at $15,15,14$, and $21 \mathrm{nM}$, respectively, whereas the clinically used drug for $\mathrm{HIV}$ 1 zidovudine (AZT) showed less activity with the $\mathrm{IC}_{50}$ value ranging from 77 to $95 \mathrm{nM}$ (Zhang et al. 2017).

Antifungal activities

Antifungal activities of arylnaphthalene lactones are summarized in Table 3. In 2003, the Gertsch group validated antifungal properties of water, dichloromethane, and $\mathrm{MeOH}$ extracts of Phyllanthus piscatorum. While the extracts did not exhibit an inhibitory effect against gram-positive bacterial strains of Pseudomonas aeruginosa, Bacillus cereus, Staphylococcus aureus, and Staphylococcus epidermis, they showed significant activity against Aspergillus fumigatus, Aspergillus flavus, and Candida albicans (Gertsch et al. 2004). In a subsequent study, the dichloromethane extract of $P$. piscatorum resulted in the activity of arylnaphthalene lactone justicidin B (1) and piscatorin (10) when tested against A. flavus, A. fumigatus, and $C$. albicans. The most active compound was justicidin B (1) with MIC values ranging from 1 to $16 \mu \mathrm{g} / \mathrm{mL}$; however, showing a higher concentration of $128 \mu \mathrm{g} / \mathrm{mL}$ against Blastoschizomyces capitatus and Cryptococcus neoformans neoformans (Gertsch et al. 2003). Bioassayguided fractionation of the leaf extract of $P$. myrtifolius led to the isolation of seven lignans, namely, phyllamyricin C (12), retrojusticidin B (91), phyllamyricin A (93), phyllamyricin F, justicidin B (1),

Table 3 Antifungal activities of arylnaphthalene lignan lactones

\begin{tabular}{lllll}
\hline Compound & \multicolumn{2}{l}{ MIC $[\mu \mathrm{g} / \mathrm{mL}]$} & & \\
\cline { 2 - 5 } & Fusarium oxysporum & Aspergillus fumigatus & Candida albicans & Aspergillus flavus \\
\hline Retrojusticidin B (91) & 16 & - & - & - \\
Phyllamyricin A (93) & 32 & - & - & - \\
Phyllamyricin C (12) & 4 & - & - & - \\
Phyllamyricin E (14) & 16 & - & - & $\geq 25$ \\
Piscatorin (10) & 16 & $\geq 3$ & $\geq 8$ & $\geq 16$ \\
Justicidin B (1) & 8 & $\geq 1$ & $\geq 4$ & \\
\hline
\end{tabular}

MIC, minimum inhibitory concentration 
phyllamyricin E (14), and piscatorin (10). Their activities were validated using the susceptibility test and conidial germination inhibition assay. Phyllamyricin A (93), phyllamyricin E (14), justicidin B (1), and phyllamyricin $\mathrm{F}$ exhibited strong inhibition against Fusarium oxysporum ATCC 44,187 with an average inhibition zone of $62-68 \%(1000 \mu \mathrm{g} / \mathrm{mL})$. In addition, phyllamyricin $\mathrm{C}$ (12) showed the most significant antifungal activity with MIC and minimum fungicidal concentration values of 4.0 and $62.5 \mu \mathrm{g} /$ $\mathrm{mL}$, respectively, and the seven lignans inhibited conidia germination of $F$. oxysporum in a concentration-dependent manner (Windayani et al. 2014).

\section{Neuroprotective activities}

Justicidin A (26) was investigated for neuroprotective activities in a cellular model of Alzheimer's disease induced by amyloid beta $(\mathrm{A} \beta)_{25-35}$ in SH-SY5Y cells. $\mathrm{A} \beta_{25-35}$-induced hyperphosphorylation of tau and okadaic acid-induced hyperphosphorylation were significantly inhibited by pre-treatment with justicidin A at 62.5, 125, and $250 \mathrm{nM}$ in a dose-dependent manner. At the same concentration, justicidin A produced a significant level of decrease in the phosphorylation of glycogen synthase kinase-3beta (GSK-3 $\beta$ ) and stimulated the phosphorylation of AMP-activated protein kinase (AMPK). In addition, treatment with justicidin $\mathrm{A}$, resulted in an increase in the level of the LC3 II/I ratio. These results show that justicidin $\mathrm{A}$ induced autophagy and inhibited neuronal cell death through reducing hyperphosphorylation of tau (Gu et al. 2016).

\section{Anti-inflammatory activities}

Prieto et al. (1996) reported for the first time the antiinflammatory activity of an $\mathrm{MeOH}$ extract of Haplophyllum hispanicum. The edema of carrageenaninduced paw and TPA-induced ear in mice showed $50 \%$ and $37 \%$ inhibition at $0.5 \mathrm{mg} /$ ear. Following the guided bioassay, the active compound diphyllin acetylapioside (43) was isolated and showed a significant inhibitory effect against TPA-induced inflammation in mice with a $50 \%$ inhibitory dose $\left(\mathrm{ID}_{50}\right)$ value at $0.27 \mu \mathrm{Mol} /$ ear (Prieto et al. 1996). In a further investigation, the same authors validated the antiinflammatory effects on eicosanoid metabolism using an HPLC-DAD-based method. Diphyllin acetylapioside showed complete inhibition of 5-lipoxygenase activity at $50 \mu \mathrm{M}$ and exhibited strong inhibitory effects against $\mathrm{LTB}_{4}$ and 5-hydroxy-6,8,11,14eicosateraenoic acid with $\mathrm{IC}_{50}$ values of 0.6 and $0.7 \mu \mathrm{M}$, respectively; however, diphyllin apioside (41) did not exhibit any effect on 5-lipoxygenase (Prieto et al. 2002). Five lignans isolated from the root of Acanthopanax chiisanenesis were examined for their effect on the production of TPA-induced $\mathrm{PGE}_{2}$ in rat peritoneal macrophages to elucidate their mechanism of action. Taiwanin C (2) exhibited the most significant inhibitory effect with an $\mathrm{IC}_{50}$ value at $0.12 \mu \mathrm{M}$ but showed no effect on the expression of TPA-induced COX-2 protein. However, with $\mathrm{IC}_{50}$ values at 1.06 and $9.31 \mu \mathrm{M}$, taiwanin $\mathrm{C}$ inhibited the activities of separated COX-1 and COX-2. These results suggest that taiwanin $\mathrm{C}$ (2) inhibits $\mathrm{PGE}_{2}$ production by directly inhibiting COX enzymatic activity (Ban et al. 2002). Three arylnaphthalide lignans from Phyllanthus polyphyllus displayed antiinflammatory effects as measured by NO, TNF- $\alpha$, and interleukin (IL-12). Justicidin B (1) exhibited the highest $\mathrm{IC}_{50}$ values of NO production from LPS/IFN$\gamma$-stimulated peritoneal macrophages at $12.5 \mu \mathrm{M}$ followed by phyllamyricin $\mathrm{C}(\mathbf{1 2})$ at $25 \mu \mathrm{M}$, and dipyllin (18) at $50 \mu \mathrm{M}$ and $100 \mu \mathrm{M}$ showing inhibition percentages of $99 \%, 99 \%$, and $64 \%$, respectively. In addition, they showed significant inhibition of IL-12 and TNF- $\alpha$ production with $\mathrm{IC}_{50}$ values ranging from 12.5 to $100 \mu \mathrm{M}$ (Rao et al. 2006).

\section{Conclusion}

Natural arylnaphthalene lactones have a $7^{\prime}$-phenyl naphthalene lactone skeleton in which the phenyl ring and naphthalene ring are polyhydroxylated, which are further transformed to methyl ethers or dioxolane. The hydroxy group, especially that at the $\mathrm{C} 7$ position is commonly conjugated with a variety of sugars to present mono-, di-, and triglycoside metabolites. Structurally, they can be classified into Type I and Type II arylnaphthalene lactones by the cis and trans relationship of lactone carbonyl and the aryl substituents. More than a hundred natural arylnaphthalene lactones have been reported from a wide range of natural sources such as Acanthaceae, Phyllanthaceae, and Schisandraceae.

Arylnaphthalene lactones exhibit various significant biological activities, which have been 
summarized here based on their pharmacological activity. Although all the natural compounds were not fully evaluated, some results such as antiproliferative and antiviral activity could give insights for drug discovery. In fact, several arylnaphthalene lactones such as diphyllin and daurinol have been investigated as anticancer drug candidates with impressive in vitro and in vivo antiproliferative activity. More recently, daurinol was investigated extensively as an antiautoimmune arthritis drug candidate. In the realm of medicinal chemistry, identifying new and valuable scaffolds is always of great interest. Thus, arylnaphthalene lactones attract considerable attention owing to their unique structural features, which include a relative rigid structure, no stereogenic center, and more than nine potential derivatizable sites. The unique structural features and promising pharmacological activities of arylnaphthalene lactones provide great prospects for future drug discovery.

Author contributions DS planned this manuscript. SP and SK searched the reported publications related to this review article. DS and SP wrote the draft of the manuscript, and SP prepared all figures. All authors approved the manuscript in its final form for publication.

Funding This work was supported by grants from the Science Research Program through the National Research Foundation of Korea (NRF), Grant No. NRF-2018M3A9C8024360 and NRF2020R1A6A1A03043708.

\section{Compliance with ethical standards}

Conflict of interest The authors declare that the research was conducted in the absence of any commercial or financial relationships that could be construed as a potential conflict of interest.

Open Access This article is licensed under a Creative Commons Attribution 4.0 International License, which permits use, sharing, adaptation, distribution and reproduction in any medium or format, as long as you give appropriate credit to the original author(s) and the source, provide a link to the Creative Commons licence, and indicate if changes were made. The images or other third party material in this article are included in the article's Creative Commons licence, unless indicated otherwise in a credit line to the material. If material is not included in the article's Creative Commons licence and your intended use is not permitted by statutory regulation or exceeds the permitted use, you will need to obtain permission directly from the copyright holder. To view a copy of this licence, visit http://creativecommons.org/licenses/by/4.0/.

\section{References}

Al-Abed Y, Sabri S, Zarga MA, Shah Z, Atta-ur-Rahman (1990) Chemical constituents of the flora of jordan, part V-B. Three new arylnaphthalene lignan glucosides from Haplophyllum buxbaumii. J Nat Prod 53(5):1152-1161

Anastas PT, Stevenson R (1991) Synthesis of natural lignan arylnaphthalene lactones, daurinol and retrochinensin. J Nat Prod 54:1687-1691

Anjaneyulu ASR, Ramaiah PA, Row LR, Venkateswarlu R, Pelter A, Ward RS (1981) New lignans from the heartwood of cleistanthus collinus. Tetrahedron 37(21):3641-3652

Arnold BJ, Mellows SM, Sammes PG (1973) Photochemical reactions. Part I. A new route to tetradehydropodophyllotoxin, taiwanin E, and related compounds. J Chem Soc Perkin Trans 1:1266-1270

Asano J, Chiba K, Tada M, Yoshii T (1996) Antiviral activity of lignans and their glycosides from Justicia procumbens. Phytochem 42(3):713-717

Ban HS, Lee S, Kim YP, Yamaki K, Shin KH, Ohuchi K (2002) Inhibition of prostaglandin $\mathrm{E}(2)$ production by taiwanin $\mathrm{C}$ isolated from the root of Acanthopanax chiisanensis and the mechanism of action. Biochem Pharmacol 64(9):1345-1354

Batsuren D, Batirov ÉK, Malikov VM, Zemlyanskii VN, Yagudaev MR (1981) Arylnaphthalene lignans of Haplophyllum dauricum. The structure of daurinol. Chem Nat Compd 17(3):223-225

Block E, Stevenson R (1971) Synthesis of model aryltetralin ligands: adducts of o-methylbenzophenone with unsymmetrical dienophiles. J Chem Soc D Chem Commun. https://doi.org/10.1039/C29710000711

Block E, Stevenson R (1973) The irradiation of 2-methylbenzophenone in the presence of dienophiles. J Chem Soc Perkin Trans 1:308-313

Borges LDC, Negrao-Neto R, Pamplona S et al (2018) Antiinflammatory and antinociceptive studies of hydroalcoholic extract from the leaves of Phyllanthus brasiliensis (Aubl.) Poir. and isolation of 5-O-b-D-glucopyranosyljusticidinB and six other lignans. Molecules 23:941

Brown D, Stevenson R (1964) Action of N, N-dicyclohexylcarbodiimide on phenylpropiolic acids: synthesis of dehydro-otobain. Tetrahedron Lett 5:3213-3216

Brown D, Stevenson R (1965) Synthesis of dehydrootobain. J Org Chem 30:1759-1763

Bucher JE (1910) The acids of the phenylpropiolic series and their condensation to naphthalene derivatives. J Am Chem Soc 32:212-221

Burden RS, Crombie L, Whiting DA (1969) The extractives of Heliopsis scabra constitution of two new lignans. J Chem Soc C 32(5):693-701

Chang CW, Lin MT, Lee SS, Liu KCSC, Hsu FL, Lin JY (1995) Differential inhibition of reverse transcriptase and cellular DNA polymerase- $\alpha$ activities by lignans isolated from Chinese herbs, Phyllanthus myrtifolius Moon, and tannins from Lonicera japonica Thunb and Castanopsis hystrix. Antiviral Res 27(4):367-374

Chang WL, Chiu LW, Lai JH et al (2003) Immunosuppressive flavones and lignans from Bupleurum scorzonerifolium. Phytochem 64:1375-1379 
Chen CC, Hsin WC, Ko FN, Huang YL, Ou JC, Teng CM (1996) Antiplatelet arylnaphthalide lignans from Justicia procumbens. J Nat Prod 59(12):1149-1150

Chen B, Liu Y, Feng C, Li BG, Zhang GL (2002) Two new arylnaphthalene lignan glycosides from Mananthes patentiflora. Chin Chem Lett 13(10):959-962

Chen H, Liu P, Zhang T, Gao Y, Zhang Y, Shen X, Li X, Shen W (2018) Effects of diphyllin as a novel V-ATPase inhibitor on TE-1 and ECA-109 cells. Oncol Rep 39(3):921-928

Cow C, Leung C, Charlton JL (2000) Antiviral activity of arylnaphthalene and aryldihydronaphthalene lignans. Can J Chem 78(5):553-561

Day SH, Chiu NY, Won SJ, Lin CN (1999) Cytotoxic lignans of Justicia ciliata. J Nat Prod 62(7):1056-1058

Day SH, Chiu NY, Tsao LT, Wang JP, Lin CN (2000) New lignan glycosides with potent antiinflammatory effect, isolated from Justicia ciliata. J Nat Prod 63(11):1560-1562

Day SH, Lin YC, Tsai ML, Tsao LT, Ko HH, Chung MI, Lee JC, Wang JP, Won SJ, Lin CN (2002) Potent cytotoxic lignans from Justicia procumbens and their effects on nitric oxide and tumor necrosis factor- $\alpha$ production in mouse macrophages. J Nat Prod 65(3):379-381

De Silva SO, Denis CS, Rodrigo R (1980) A regiocontrolled synthesis of some arylnaphthalide lignans. J Chem Soc Chem Commun 995-997

Evcim U, Gōzler B, Freyer AJ, Shamma M (1986) Haplomyrtin and (-)-haplomyrfolin: two lignans from haplophyllum myrtifolium. Phytochem 25(8):1949-1951

Gertsch J, Tobler RT, Brun R, Sticher O, Heilmann J (2003) Antifungal, antiprotozoal, cytotoxic and piscicidal properties of Justicidin B and a new arylnaphthalide lignan from Phyllanthus piscatorum. Planta Med 69(05):420-424

Gertsch J, Niomawë Gertsch-Roost K, Sticher O (2004) Phyllanthus piscatorum, ethnopharmacological studies on a women's medicinal plant of the Yanomamï Amerindians. J Ethnopharmacol 91(2):181-188

Ghosal S, Banerjee S (1979) Synthesis of retrochinensin; a new naturally occurring 4-aryl-2,3-naphthalide lignan. J Chem Soc, Chem Commun 4:165-166

Ghosal S, Chauhan RPS, Srivastava RS (1974) Two new aryl naphthalide lignans from Polygala chinensis. Phytochem 13(9):1933-1936

Gözler T, Gözler B, Patra A, Leet JE, Freyer AJ, Shamma M (1984) Konyanin: a new lignan from hapuophyllum vulcaniclm. Tetrahedron 40(7):1145-1150

Gözler B, Gözler T, Sağlam H, Hesse M (1996) Minor lignans from haplophyllum cappadocicum. Phytochemistry 42(3):689-693

Gu MY, Kim J, Yang HO (2016) The neuroprotective effects of justicidin A on amyloid beta25-35-induced neuronal cell death through inhibition of tau hyperphosphorylation and induction of autophagy in SH-SY5Y Cells. Neurochem Res 41(6):1458-1467

Hayat F, Park SH, Choi NS, Lee J, Park SJ, Shin D (2015a) Synthesis and anticancer activity of 4-aza-daurinol derivatives. Arch Pharm Res 38(11):1975-1982

Hayat F, Kang L, Lee CY, Shin D (2015b) Synthesis of arylnaphthalene lignan lactone using benzoin condensation, intramolecular thermal cyclization and Suzuki coupling. Tetrahedron 71:2945-2950
He XL, Zhang P, Dong XZ, Yang MH, Chen SL, Bi MG (2012) JR6, a new compound isolated from Justicia procumbens, induces apoptosis in human bladder cancer EJ cells through the caspase-dependent pathway. J Ethnopharmacol 144(2):284-292

Hemmati S, Seradj H (2016) Justicidin B: a promising bioactive lignan. Molecules 21(7):820

Hesse M, Gozler B, Arar G, Gozler T (1992) Isodaurinol, an arylnaphthalene lignan from Haplophyllum cappadocicum. Phytochem 31(7):2473-2475

Holmes TL, Stevenson R (1971) Arylnaphthalene lignans: synthesis of helioxanthin. J. Chem. Soc. C Org 2091-2094

Innocenti G, Puricelli L, Piacente S, Caniato R, Filippini R, Cappelletti EM (2002) Patavine, a new arylnaphthalene lignan glycoside from shoot cultures of Haplophyllum patavinum. Chem Pharm Bull 50(6):844-846

Jin H, Yin HL, Liu SJ et al (2014) Cytotoxic activity of lignans from Justicia procumbens. Fitoterapia 94:70-76

Jin H, Yang S, Dong JX (2017) New lignan glycosides from Justicia procumbens. J Asian Nat Prod Res 19:1-8

Kang K, Oh SH, Yun JH, Jho EH, Kang JH, Batsuren D., Tunsag J, Park KH, Kim M, Nho CW (2011) A novel topoisomerase inhibitor, daurinol, suppresses growth of HCT116 cells with low hematological toxicity compared to etoposide. Neoplasia (New York, N.Y.) 13(11):1043-1057

Kavitha J, Gopalaiah K, Rajasekhar D et al (2003) Juspurpurin, an Unusual Secolignan Glycoside from Justicia purpurea. J Nat Prod 66:1113-1115

Khalid SA, Waterman PG (1981) Alkaloid, lignan and flavonoid constituents of Haplophyllum tuberculatum from Sudan. Planta Med 43(10):148-152

Kuo Y-H, Wu T-R, Cheng M-C, Wang Y (1990) Five new compounds from the heartwood of Juniperus formosana Hayata. Chem Pharm Bull 38(12):3195-3201

Lee SS, Lin MT, Liu CL, Lin YY, Karin CS, Liu C (1996) Six lignans from Phyllanthus myrtifolius. J Nat Prod 59(11):1061-1065

Li Y, Fu L, Yeo H, Zhu JL, Chou CK, Kou YH, Yeh SF, Gullen E, Austin D, Cheng YC (2005) Inhibition of Hepatitis B virus gene expression and replication by helioxanthin and its derivative. Antivir Chem Chemother 16(3):193-201

Li S, Liang Z, Li J, Zhang X, Zheng R, Zhao C (2020) Update on naturally occurring novel arylnaphthalenes from plants. Phytochem Rev 19:337-403

Lin MT, Lee SS, Liu KCSC (1995) Phyllamyricins A-C, three novel lignans from Phyllanthus myrtifolius. J Nat Prod 58(2):244-249

Liu G, Wu J, Si J, Wang J, Yang M (2008a) Complete assignments of $1 \mathrm{H}$ and 13C NMR data for three new arylnaphthalene lignan from Justicia procumbens. Magn Reson Chem 46(3):283-286

Liu Y, Zhang TT, Zhou JS et al (2008b) Three new arylnaphthalide lignans from the aerial parts of Bupleurum marginatum WALL. ex DC. Helv Chim Acta 91:2316-2320

Liu Y, Young K, Rakotondraibe LH et al (2015) Antiproliferative compounds from Cleistanthus boivinianus from the madagascar dry forest. J Nat Prod 78:1543-1547

Luo J, Hu Y, Kong W, Yang M (2014) Evaluation and structureactivity relationship analysis of a new series of 
arylnaphthalene lignans as potential anti-tumor agents. PLoS ONE 9(3):e93516

Luo J, Qin J, Fu Y, Zhang S, Zhang X, Yang M (2018) 6'Hydroxy Justicidin B triggers a critical imbalance in $\mathrm{Ca}(2+)$ homeostasis and mitochondrion-dependent cell death in human leukemia K562 cells. Front Pharmacol 9:601-601

Ma JX, Lan MS, Qu SJ et al (2012) Arylnaphthalene lignan glycosides and other constituents from Phyllanthus reticulatus. J Asian Nat Prod Res 14:1073-1077

Maclean I, Stevenson R (1966) Synthesis of ( \pm )-otobain. J Chem Soc C: Org 1717-1719

Michael A, Bucher JE (1895) Ueber die einwirkung von essigsäureanhydrid auf säuren der acetylenreihe. Ber Deutsch Chem Ges 28:2511-2512

Mohagheghzadeh A, Schmidt TJ, Alfermann AW (2002) Arylnaphthalene lignans from in vitro cultures of Linum austriacum. J Nat Prod 65:69-71

Navarro E, Alonso SJ, Trujillo J, Jorge E, Pérez C (2001) General behavior, toxicity, and cytotoxic activity of Elenoside, a lignan from Justicia hyssopifolia. J Nat Prod 64(1):134-135

Novelo M, Cruz JG, Hernández L, Pereda-Miranda R, Chai H, Mar W, Pezzuto JM (1993) Cytotoxic constituents from Hyptis verticillata. J Nat Prod 56(10):1728-1736

Nukul GS, Abu Zarga MH, Sabri SS, Al-Eisawi DM (1987) Chemical constituents of the Flora of Jordan, Part III. Mono-O-acetyl Diphyllin Apioside, a new arylnaphthalene lignan from Haplophyllum buxbaumii. J Nat Prod 50(4):748-750

Ohta K, Munakata K (1970) Justicidin C and D, the 1-methoxy2,3-naphthalide lignans, isolated from Justicia procumbens L. Tetrahedron Lett 11(12):923-925

Pandey DP, Nautiyal DP, Rather MA et al (2011) A new arylnaphthalide lignan from Justicia prostrata Gamble. Asian J Chem 23:2125-2127

Park JE, Lee J, Seo SY, Shin D (2014) Regioselective route for arylnaphthalene lactones: convenient synthesis of taiwanin C, justicidin E, and daurinol. Tetrahedron Lett 55:818-820

Park MJ, Moon SJ, Lee EJ, Kim EK, Beak JA, Kim SY, Jung KA, Lee SH, Choi JW, Kim DS, Min JK, Park SH, Shin DY, Cho ML (2019) Daurinol Attenuates Autoimmune Arthritis via Stabilization of Nrp1-PTEN-Foxp3 Signaling in Regulatory T Cells Front Immunol, 10; article 1526

Park S, Kim JH, Kim SH, Shin D (2020) Transition metalmediated annulation approaches for synthesis of arylnaphthalene lignan lactones. Chem, Front. https://doi.org/ 10.3389/fchem.2020.00628

Plaumann HP, Smith JG, Rodrigo R (1980) Potential isobenzofurans: their use in the synthesis of naturally occurring 1-arylnaphthalide lignans. J Chem Soc, Chem Commun $8: 354-355$

Prieto JM, Recio MC, Giner RM, Máñez S, Massmanian A, Waterman PG, Ríos JL (1996) Topical Anti-Inflammatory Lignans from Haplophyllum hispanicum. Z Naturforsch 51c:618-622

Prieto JM, Giner RM, Recio Mf MC, Schinella G, Manez S, Rios JL (2002) Diphyllin acetylapioside, a 5-lipoxygenase inhibitor from Haplophyllum hispanicum. Planta Med 68(4):359-360
Rajasekhar D, Subbaraju GV (2000) Jusmicranthin, a new arylnaphthalide lignan from Justicia neesii. Fitoterapia 71(5):598-599

Ramesh C, Ravindranath N, Ram TS, Das B (2003) Arylnaphthalide lignans from Cleistanthus collinus. Chem Pharm Bull 51(11):1299-1300

Rao YK, Fang SH, Tzeng YM (2006) Anti-inflammatory activities of constituents isolated from Phyllanthus polyphyllus. J Ethnopharmacol 103(2):181-186

Ren Y, Lantvit DD, Deng Y, Kanagasabai R, Gallucci JC, Ninh TN, Chai HB, Soejarto DD, Fuchs JR, Yalowich JC, Yu J, Swanson SM, Kinghorn AD (2014) Potent cytotoxic arylnaphthalene lignan lactones from Phyllanthus poilanei. J Nat Prod 77(6):1494-1504

Rezanka T, Rezanka P, Sigler K (2009) Glycosides of arylnaphthalene lignans from Acanthus mollis having axial chirality. Phytochem 70:1049-1054

Sastry KV, Rao EV (1983) Isolation and Structure of Cleistanthoside A. Planta Med 47(04):227-229

Satyanarayana V, Krupadanam GL, Srimannarayana G (1991) A butyrolactone lignan disaccharide from Flacourtia ramontchi. Phytochem 30(3):1026-1029

Shen W, Zou X, Chen M, Liu P, Shen Y, Huang S, Guo H, Zhang L (2011) Effects of diphyllin as a novel V-ATPase inhibitor on gastric adenocarcinoma. Eur J Pharmacol 667(1):330-338

Song YN, Zhang HL, Chang CJ, Bollag DM (1994) Cytotoxic cyclolignans from Koelreuteria henryi. J Nat Prod 57(12):1670-1674

Stevenson R, Block E (1971) Lignan lactones. Synthesis of ( \pm )collinusin and justicidin B. J Org Chem 36:3453-3455

Stevenson R, Holmes TL (1971) Arylnaphthalene lignans. Synthesis of justicidin E, taiwanin C, dehydrodimethylconidendrin, and dehydrodimethylretrodendrin. J Org Chem 36:3450-3453

Stevenson R, Weber JV (1989) Improved methods of synthesis of lignan arylnaphthalene lactones via arylpropargyl arylpropiolate esters. J Nat Prod 52:367-375

Stevenson R, Weber JV (1991) Synthesis of lignan aryldihydronaphthalene lactones by cyclization of cinnamyl arylpropiolate esters-revised structure of beta apopolycamatin. J Nat Prod 54:310-314

Susplugas S, Hung NV, Bignon J, Thoison O, Kruczynski A, Sévenet T, Guéritte F (2005) Cytotoxic arylnaphthalene lignans from a Vietnamese Acanthaceae, Justicia patentiflora. J Nat Prod 68(5):734-738

Teponno RB, Kusari S, Spiteller M (2016) Recent advances in research on lignans and neolignans. Nat Prod Rep 33:1044-1092

Tian JM, Hao XJ, He HP (2006a) A new lignan and four new lignan glycosides from Mananthes patentiflora. Helv Chim Acta 89:291-298

Tian J, Hao X, He H (2006b) A new lignan and four new lignan glycosides from Mananthes patentiflora. Helvetica 89(2):291-298

Tian JM, He HP, Di YT, Yang XW, Gao ZL, Hao XJ (2008) Three new lignan glycosides from Mananthes patentiflora. J Asian Nat Prod Res 10(3):228-232

Tuchinda P, Kumkao A, Pohmakotr M, Sophasan S, Santisuk T, Reutrakul V (2006) Cytotoxic arylnaphthalide lignan 
glycosides from the aerial parts of Phyllanthus taxodiifolius. Planta Med 72(1):60-62

Tuchinda P, Kornsakulkarn J, Pohmakotr M et al (2008) Dichapetalin-type triterpenoids and lignans from the aerial parts of Phyllanthus acutissima. J Nat Prod 71:655-663

Wada K, Munakata K (1970) (-) Parabenzlactone, a new piperolignanolide isolated from Parabenzoin trilobum nakai. Tetrahedron Lett 11(23):2017-2019

Wang CLJ, Ripka WC (1983) Total synthesis of (. + -.)-justicidin P. A new lignan lactone from Justicia extensa. J Org Chem 48(15):2555-2557

Wang HC, Tseng YH, Wu HR, Chu FH, Kuo YH, Wang SY (2014) Anti-proliferation effect on human breast cancer cells via inhibition of $\mathrm{pRb}$ phosphorylation by taiwanin $\mathrm{E}$ isolated from Eleutherococcus trifoliatus. Nat Prod Commun 9(9):1303-1306

Wei CY, Wang SW, Ye JW et al (2018) New anti-inflammatory aporphine and lignan derivatives from the root wood of Hernandia nymphaeifolia. Molecules 23:2286

Weng JR, Ko HH, Yeh TL, Lin HC, Lin CN (2004) Two new arylnaphthalide lignans and antiplatelet constituents from Justicia procumbens. Arch Pharm 337(4):207-212

Windayani N, Rukayadi Y, Hakim EH, Ruslan K, Syah YM (2014) Antifungal activity of lignans isolated from Phyllanthus myrtifolius Moon. against Fusarium oxysporum. Phytochem 12:33-39

Woo JK, Jung HJ, Park JY, Kang JH, Lee BI, Shin D, Nho CW, Cho SY, Seong JK, Oh SH (2017) Daurinol blocks breast and lung cancer metastasis and development by inhibition of focal adhesion kinase (FAK). Oncotarget 8(34):57058-57071

Wu SJ, Wu TS (2006) Cytotoxic arylnaphthalene Lignans from Phyllanthus oligospermus. Chem Pharm Bull 54(8):1223-1225

Wu WW, Liao LP, Wang XJ et al (2012) A new lignan glycoside procumbenoside E from Justicia procumbens L. Chin J Pharm 43:669-672
Yamamoto Y, Mori S, Shibuya M (2015) A combined transition-metal-catalyzed and photopromoted process: synthesis of 2,3-fused 4-Phenylnaphthalen-1-yl carboxylates from 1,7-diaryl-1,6-diynes. Chem Eur J 21:9093-9100

Yang M, Wu J, Cheng F, Zhou Y (2006) Complete assignments of $1 \mathrm{H}$ and 13C NMR data for seven arylnaphthalide lignans from Justicia procumbens. Magn Reson Chem 44(7):727-730

Yeo H, Li Y, Fu L, Zhu JL, Gullen EA, Dutschman GE, Lee Y, Chung R, Huang ES, Austin DJ, Cheng YC (2005) Synthesis and antiviral activity of Helioxanthin analogues. J Med Chem 48(2):534-546

Yu Z, Dan W, Jie H, Li Z (2010) Synthesis and bioevaluation of novel analogues of justicidin A. Med Chem Res 19(1):71-76

Yu S, Lv JJ, Zhao JQ et al (2016) New cytotoxic lignan glycosides from Phyllanthus glaucus. Nat Prod Res 30:419-425

Zhang ZZ, Ownby S, Wang P et al (2010) New phenolic compounds from Liatris elegans. Nat Prod Res 24:1079-1085

Zhang Z, Ma J, Zhu L, Zhao Y (2014) Synthesis and identification of cytotoxic diphyllin glycosides as vacuolar $\mathrm{H}+$ ATPase inhibitors. Eur J Med Chem 82:466-471

Zhang HJ, Rumschlag-Booms E, Guan YF, Liu KL, Wang DY, Li WF, Nguyen VH, Cuong NM, Soejarto DD, Fong HHS, Rong L (2017) Anti-HIV diphyllin glycosides from Justicia gendarussa. Phytochem 136:94-100

Zheng CJ, Zhang XW, Han T et al (2014) Anti-inflammatory and anti-osteoporotic lignans from Vitex negundo seeds. Fitoterapia 93:31-38

Publisher's Note Springer Nature remains neutral with regard to jurisdictional claims in published maps and institutional affiliations. 